ESAIM: COCV 27 (2021) 87

https://doi.org/10.1051/cocv/2021082
ESAIM: Control, Optimisation and Calculus of Variations

www.esaim-cocv.org

\title{
INVERSE POTENTIAL PROBLEMS IN DIVERGENCE FORM FOR MEASURES IN THE PLANE*,**
}

\author{
Laurent Baratchart ${ }^{1, * * *}$, Cristóbal Villalobos Guillén ${ }^{2}$ \\ AND Douglas P. HaRdin ${ }^{3}$
}

\begin{abstract}
We study inverse potential problems with source term the divergence of some unknown $\left(\mathbb{R}^{3}\right.$-valued) measure supported in a plane; e.g., inverse magnetization problems for thin plates. We investigate methods for recovering a magnetization $\boldsymbol{\mu}$ by penalizing the measure-theoretic total variation norm $\|\boldsymbol{\mu}\|_{T V}$, and appealing to the decomposition of divergence-free measures in the plane as superpositions of unit tangent vector fields on rectifiable Jordan curves. In particular, we prove for magnetizations supported in a plane that $T V$-regularization schemes always have a unique minimizer, even in the presence of noise. It is further shown that $T V$-norm minimization (among magnetizations generating the same field) uniquely recovers planar magnetizations in the following two cases: (i) when the magnetization is carried by a collection of sufficiently separated line segments and a set that is purely 1-unrectifiable; (ii) when a superset of the support is tree-like. We note that such magnetizations can be recovered via $T V$-regularization schemes in the zero noise limit by taking the regularization parameter to zero. This suggests definitions of sparsity in the present infinite dimensional context, that generate results akin to compressed sensing.
\end{abstract}

Mathematics Subject Classification. 31B20, 49N45, 49Q20, 86A22.

Received March 29, 2021. Accepted July 14, 2021.

\section{INTRODUCTION}

This paper considers inverse potential problems with source term in divergence form, in connection with the structure of 2-D divergence-free measures in the plane. A typical application, and the main motivation of the authors to carry out this research, is to inverse magnetization problems on thin plates. When magnetizations are modelled by $\mathbb{R}^{3}$-valued measures supported on a set $S$ (in the thin plate case $S \subset \mathbb{R}^{2}$ ), the inverse magnetization problem consists in recovering such a measure, say $\boldsymbol{\mu}$, from knowledge of the magnetic field $\mathbf{b}(\boldsymbol{\mu})$ that it generates, see Section 1.1 for details. Magnetizations supported in a plane generate the zero magnetic field if and only if they are tangent to that plane and divergence-free there (see Lems 2.1 and 2.2). Thus, the kernel of the

\footnotetext{
* The research of the third author was supported, in part, by the U. S. National Science Foundation under grant DMS-1521749.

** The research of the second author was supported, in part, by a grant from the Fondation mathématique Jacques Hadamard.

Keywords and phrases: Planar divergence free measures, purely 1-unrectifiable sets, inverse potential problems in divergence form, thin plate magnetizations, sparse recovery, total variation regularization.

1 Projet FACTAS, INRIA, 2004 route des Lucioles, BP 93, Sophia-Antipolis, 06902 Cedex, France.

2 CMAP, Ecole Polytechnique, route de Saclay, 91128 Palaiseau Cedex, France.

3 Department of Mathematics, Vanderbilt University, Nashville, TN 37240, USA.

*** Corresponding author: laurent.baratchart@inria.fr
} 
forward operator mapping $\boldsymbol{\mu}$ to $\mathbf{b}(\boldsymbol{\mu})$ consists precisely of planar divergence-free measures in this case. Now, a 2-D divergence-free measure $\boldsymbol{\nu}$ in the plane can be decomposed as a superposition of elementary "loops"; i.e., contour integrations along rectifiable Jordan curves, in such a way that the Radon-Nykodim derivative $\mathrm{d} \boldsymbol{\nu} / \mathrm{d}|\boldsymbol{\nu}|(x)$ is essentially the unit tangent to any of these curves through $x,(c f$. Thm. 2.8). This result, which is more precise (though limited to the planar case) than the general decomposition theorem for solenoids given in [28], gives us insight on the structure of the kernel of the forward operator, enabling us to give sufficient conditions for a magnetization to be $T V$-minimal on $S$; i.e., the magnetization has minimum total variation among those magnetizations supported on $S$ that generate the same field. When a $T V$-minimal magnetization on $S$ is unique among magnetizations generating the same field, we call it strictly $T V$-minimal on $S$. By standard regularization theory, strictly $T V$-minimal magnetizations can in principle be recovered by solving a sequence of minimization problems for the so-called regularizing functional, which is the sum of the quadratic residuals and a penalty term consisting of the product of a regularization parameter $\lambda>0$ and the total variation of the unknown measure, see (1.4). Specifically, any sequence of minimizers of the regularizing functional converges weak-* to the strictly $T V$-minimal measure generating the data (when it exists), as the regularizing parameter and the noise tend jointly to zero in a suitable manner, see e.g. [11]. In short: regularizing schemes that penalize the total variation are consistent to recover strictly $T V$-minimal magnetizations, and thus, any assumption ensuring strict $T V$-minimality gives rise to a consistency result. For the larger class of magnetizations supported on a slender set $S$ (see Sect. 1.1 for a definition), such a consistency result is obtained in Theorem 2.6 of [7] by showing, using results from [28], that magnetizations supported on a purely 1-unrectifiable set are strictly $T V$-minimal. Specializing to the case of planar $S$ and appealing to the above-mentioned loop decomposition, we shall obtain more general conditions: for instance magnetizations carried by the union of a purely 1-unrectifiable set and a collection of sufficiently separated line segments are strictly $T V$-minimal, see Corollary 3.4 and Theorem 3.2.

The results just mentioned are reminiscent of compressed sensing, where underdetermined systems of linear equations in $\mathbb{R}^{n}$ are approximately solved by minimizing the residuals while penalizing the $l^{1}$-norm. This favors the recovery of sparse solutions (i.e., solutions having a large number of zero components) when they exist, see e.g. $[9,19]$. In this connection, the gist of Theorem 2.6 in [7] and Corollary 3.4 is to define notions of "sparsity" in the present, infinite-dimensional context. Our results warrant the use of regularizing schemes that penalize the total variation (a natural analog of the $l^{1}$-norm), in order to recover magnetizations which are sparse according to such definitions.

Our second application of the loop decomposition to inverse magnetization problems on thin plates is to prove that, for each value of the regularization parameter, the minimizer of the regularizing functional is unique (Thm. 3.8). This result is important for algorithmic approaches, because it tells us that for every choice of the regularization parameter there is a unique estimate of the unknown magnetization based on the regularization scheme (1.5). It is also surprising, for in the case that a magnetization is $T V$-minimal but not strictly $T V$ minimal, one would rather expect the regularizing functional to have several minimizers, at least for small values of the regularizing parameter.

Let us stress that magnetizations supported in a plane are commonly considered in paleomagnetic studies, where thin slabs of rock are modeled by planar regions $[5,23,24,30]$. In particular, our analysis applies to this setting. It would be quite interesting to carry over the contents of the present paper to more general slender surfaces in $\mathbb{R}^{3}$ than planes, as the results could be of interest in other situations from geosciences or medical imaging. In practice, the development of numerically effective algorithms for such inverse problem raises delicate issues of discretization. These are not addressed in this paper, but will be taken up in future work.

The loop decomposition of planar divergence-free measures, which plays a fundamental role in our proofs, is hinted at on page 843 of [28]. To the best of our knowledge, it was first shown in [29] along the lines suggested by [28]. This method furnishes in addition a specific parametrization of the loops representing a given divergencefree measure. Two other proofs of the general loop decomposition of divergence-free planar measures are given in the manuscript [8], but without the above-mentioned parametrization. This parametrization is further refined in the manuscript [6], whose version of the loop decomposition appears in the present paper as Theorem 2.8, see the discussion in Section 2.3. This refinement is useful to establish Theorem 3.3, showing that measures carried by a collection of sufficiently separated line segments are strictly $T V$-minimal. 
In the rest of this introductory section, we describe the inverse magnetization problem, explain our main results and set up notation.

\subsection{Background and overview of results}

Let us first describe the inverse magnetization problem. For a closed subset $S \subset \mathbb{R}^{3}$, let $\mathcal{M}(S)$ denote the space of finite signed Borel measures supported on $S$. We use the space $\mathcal{M}(S)^{3}$ of $\mathbb{R}^{3}$-valued measures supported on $S$ to model physical magnetizations distributed on $S$, and shall often speak of "magnetization on $S^{\text {" to mean }}$ "element of $\mathcal{M}(S)^{3}$ ". For $\boldsymbol{\mu} \in \mathcal{M}(S)^{3}$, we let $|\boldsymbol{\mu}|$ denote the total variation measure of $\boldsymbol{\mu}$. The latter is a positive measure, and we put $\|\boldsymbol{\mu}\|_{T V}:=|\boldsymbol{\mu}|\left(\mathbb{R}^{3}\right)$ for the total variation of $\boldsymbol{\mu}$, see Section 1.2.

The magnetic field $\mathbf{b}(\boldsymbol{\mu})$ generated by a magnetization $\boldsymbol{\mu} \in \mathcal{M}(S)^{3}$ is defined, at a point $x$ not in the support of $\boldsymbol{\mu}$, in terms of the scalar magnetic potential $\Phi(\boldsymbol{\mu})$ by (see [22]):

$$
\mathbf{b}(\boldsymbol{\mu})(x)=-\mu_{0} \nabla \Phi(\boldsymbol{\mu})(x), \quad x \notin \operatorname{supp} \boldsymbol{\mu},
$$

where $\mu_{0}$ is the magnetic constant and $\nabla$ indicates the gradient. Here, $\Phi(\boldsymbol{\mu})(x)$ is given by

$$
\Phi(\boldsymbol{\mu})(x):=\frac{1}{4 \pi} \int \nabla_{y} \frac{1}{|x-y|} \cdot \mathrm{d} \boldsymbol{\mu}(y)=\frac{1}{4 \pi} \int \frac{x-y}{|x-y|^{3}} \cdot \mathrm{d} \boldsymbol{\mu}(y),
$$

where, for $x, y \in \mathbb{R}^{3}, x \cdot y$ and $|x|$ denote the Euclidean scalar product and norm and $\nabla_{y}$ the gradient with respect to $y$. Clearly, $\Phi(\boldsymbol{\mu})$ and the components of $\mathbf{b}(\boldsymbol{\mu})$ are harmonic functions on $\mathbb{R}^{3} \backslash S$. Moreover, formula (1.2) defines $\Phi(\boldsymbol{\mu})$ on the whole of $\mathbb{R}^{3}$ as a member of $L^{2}\left(\mathbb{R}^{3}\right)+L^{1}\left(\mathbb{R}^{3}\right)$ (see [7], Prop. 2.1) so that $\mathbf{b}(\boldsymbol{\mu})$, initially defined on $\mathbb{R}^{3} \backslash S$, extends to a $\mathbb{R}^{3}$-valued divergence-free distribution on $\mathbb{R}^{3}$. Indeed, we may write

$$
\Delta \Phi=\nabla \cdot \boldsymbol{\mu} \quad \text { and } \quad \mathbf{b}(\boldsymbol{\mu})=\mu_{0}(\boldsymbol{\mu}-\nabla \Phi(\boldsymbol{\mu})),
$$

where $\nabla \cdot \boldsymbol{\mu}$ indicates the divergence of $\boldsymbol{\mu}$. Note that (1.3) yields a Helmholtz-Hodge decomposition of $\boldsymbol{\mu}$, as the sum of a gradient and a divergence-free distribution. However, neither term is a measure in general but rather a distribution of order -1 .

The inverse magnetization problem is to recover $\boldsymbol{\mu}$ from measurements of $\mathbf{b}(\boldsymbol{\mu})$ taken on a set $Q \subset \mathbb{R}^{3} \backslash S$ which, due to the oriented nature of sensors (coils), are typically observed in one direction only, say along some unit vector $v \in \mathbb{R}^{3}$. We assume for simplicity that $v$ is the same at each measurement point. For instance, it is usually so in Scanning Magnetic Microscopy experiments (SMM) where data consist of point-wise values of the normal component of the magnetic field on a planar region not intersecting $S$, see $[23,24,30]$. Geometric conditions on $Q, S$ and $v$, ensuring that such measurements suffice to determine $\mathbf{b}(\boldsymbol{\mu})$ in the entire region $\mathbb{R}^{3} \backslash S$, are given in Lemma 2.3 of [7] and recalled when $S$ is planar in Section 3.2, for the convenience of the reader. In the remainder of this introduction, we assume that these assumptions are satisfied.

Still, the mapping $\boldsymbol{\mu} \rightarrow \mathbf{b}(\boldsymbol{\mu})$ is generally not injective, which is a major difficulty with this inverse problem. In this connection, we say that $\boldsymbol{\mu}, \boldsymbol{\nu} \in \mathcal{M}(S)^{3}$ are $S$-equivalent if $\mathbf{b}(\boldsymbol{\mu})$ and $\mathbf{b}(\boldsymbol{\nu})$ agree on $\mathbb{R}^{3} \backslash S$. A magnetization $\boldsymbol{\mu}$ is said to be $S$-silent if $\boldsymbol{\mu}$ is $S$-equivalent to the zero magnetization; i.e., if $\mathbf{b}(\boldsymbol{\mu})$ vanishes on $\mathbb{R}^{3} \backslash S$.

Since no nonzero harmonic function lies in $L^{2}\left(\mathbb{R}^{3}\right)+L^{1}\left(\mathbb{R}^{3}\right)$, it follows from (1.3) that a divergence-free magnetization is $S$-silent; here and below, the divergence is understood in the distributional sense. A partial converse is given in Theorem 2.2 of [7], namely a $S$-silent magnetization is divergence-free provided that $S$ is slender, meaning it has Lebesgue measure zero and each connected component of $\mathbb{R}^{3} \backslash S$ has infinite Lebesgue measure. The slenderness assumption is a strong one: for instance it rules out the case where $S$ is a volumic sample or a closed surface. However, it is satisfied in important special cases, for example in paleomagnetic studies, as mentioned already, or in Geomagnetism where some regions of the Earth's crust are assumed to be non-magnetic (or much less magnetic) than the others [20], or even in Electro-Encephalography where sources of primary current are often considered to lie on the surface of the encephalon (which is closed and therefore 
not slender) but their support should arguably leave out the brain stem connecting to the spinal cord (therefore the support is contained in a slender set).

In [28], Smirnov describes $\mathbb{R}^{n}$-valued divergence-free measures in $\mathbb{R}^{n}$, also known as solenoids, in terms of integrals of elementary components that are absolutely continuous with respect to 1-dimensional Hausdorff measure $\mathcal{H}^{1}$. Consequently, if $S$ is slender and $\boldsymbol{\mu} \in \mathcal{M}(S)^{3}$ is carried by a purely 1-unrectifiable set (i.e., whose intersection with any 1-rectifiable set has $\mathcal{H}^{1}$ - measure zero, see [25]), then $\boldsymbol{\mu}$ is mutually singular to every $S$-silent magnetization and so has minimum total variation amongst all magnetizations that are $S$-equivalent to $\boldsymbol{\mu}$. This observation led the authors in [7] to consider the following extremal problem involving the quantity $M_{S}(\boldsymbol{\mu})$, defined for $\boldsymbol{\mu} \in \mathcal{M}(S)^{3}$ by

$$
M_{S}(\boldsymbol{\mu}):=\inf \left\{\|\boldsymbol{\nu}\|_{T V}: \boldsymbol{\nu} \text { is } S \text {-equivalent to } \boldsymbol{\mu}\right\} .
$$

Extremal Problem 1. Given $\boldsymbol{\mu}_{0} \in \mathcal{M}(S)^{3}$, find $\boldsymbol{\mu}$ that is $S$-equivalent to $\boldsymbol{\mu}_{0}$ satisfying

$$
\|\boldsymbol{\mu}\|_{T V}=M_{S}\left(\boldsymbol{\mu}_{0}\right)
$$

A solution to Extremal Problem 1 is, by definition, $T V$-minimal on $S$, and it is strictly $T V$-minimal on $S$ if this solution is unique. When $S \subset \mathbb{R}^{3}$ is slender and $\boldsymbol{\mu}_{0} \in \mathcal{M}(S)^{3}$, we find that $\boldsymbol{\mu}_{0}$ is strictly $T V$-minimal on $S$ for the three cases listed below. Here case (a) is essentially ([7], Thm. 2.6) and a special case of Theorem 3.2 to come, while (b) is contained in Theorem 2.11 of [7] and (c) follows from Corollary 3.4 further below.

(a) there is a purely 1-unrectifiable set of full $\left|\boldsymbol{\mu}_{0}\right|$ measure;

(b) the set $S$ is a finite disjoint union of compact sets $S_{1}, \ldots S_{k}$ and

$$
\boldsymbol{\mu}_{0} L_{S_{i}}=\mathbf{u}_{i}\left|\boldsymbol{\mu}_{0}\right| L_{S_{i}}
$$

for some collection of unit vectors $\mathbf{u}_{1}, \ldots, \mathbf{u}_{k} \in \mathbb{R}^{3}$, in which case we say $\boldsymbol{\mu}_{0}$ is piecewise unidirectional;

(c) there is a set of full $\left|\boldsymbol{\mu}_{0}\right|$-measure contained in a countable union of coplanar disjoint line segments $L_{k}$ such that the distance from any $L_{k}$ to any $L_{j}, j \neq k$, is greater than or equal to $\mathcal{H}^{1}\left(L_{k}\right)$.

Corollary 3.4 also implies that (a) can be combined with (c), namely if a measure satisfies (c) and we add to it a measure on $S$ carried by a purely 1-unrectifiable set, then we get a measure which is strictly $T V$-minimal again.

Now, for $\rho$ a positive measure on $Q$, let $A: \mathcal{M}(S)^{3} \rightarrow L^{2}(Q, \rho)$ be the forward operator mapping $\boldsymbol{\mu}$ to the restriction of $\mathbf{b}(\boldsymbol{\mu}) \cdot v$ on $Q$ (see (3.13)). The measure $\rho$ does not play a significant role in what follows (e.g., it could be chosen to be Lebesgue measure on $Q$ ), but it is important for practical applications. To recover solutions of Extremal Problem 1 knowing the restriction $f$ of $\mathbf{b}\left(\boldsymbol{\mu}_{0}\right) \cdot v$ to $Q$, the theory of regularization for convex problems [11] suggests to minimize with respect to $\boldsymbol{\mu} \in \mathcal{M}(S)^{3}$ the functional

$$
\mathcal{F}_{f, \lambda}(\boldsymbol{\mu}):=\|f-A \boldsymbol{\mu}\|_{L^{2}(Q, \rho)}^{2}+\lambda\|\boldsymbol{\mu}\|_{T V}
$$

for some suitable value of the regularization parameter $\lambda>0$. That is, we consider:

Extremal Problem 2. Given $f \in L^{2}(Q)$ and $\lambda>0$, find $\boldsymbol{\mu}_{\lambda} \in \mathcal{M}(S)^{3}$ such that

$$
\mathcal{F}_{f, \lambda}\left(\boldsymbol{\mu}_{\boldsymbol{\lambda}}\right)=\inf _{\boldsymbol{\mu} \in \mathcal{M}(S)^{3}} \mathcal{F}_{f, \lambda}(\boldsymbol{\mu})
$$

When $Q$ and $S$ are positively separated, the existence of at least one minimizer in (1.5) is a consequence of the weak-* compactness of the unit ball in $\mathcal{M}(S)^{3}$ see e.g. Proposition 3.6 of [10]. Solving Extremal Problem 2 is a particular regularization scheme for the Inverse Magnetization Problem, namely one that penalizes the total variation of the unknown. 
It is standard that if $f=A \boldsymbol{\mu}_{0}$ and $\lambda_{n} \rightarrow 0$, then any subsequence of $\boldsymbol{\mu}_{\lambda_{n}}$ has a subsequence converging weak-* to a solution of Extremal Problem 1. To account for measurement noise, one usually replaces $f$ by $f_{n}=A \boldsymbol{\mu}_{0}+e_{n}$, and then the same result holds for a sequence $\boldsymbol{\mu}_{n}$ minimizing (1.4) with $f=f_{n}$ and $\lambda=\lambda_{n}$, provided that both $\lambda_{n}$ and $\left\|e_{n} \lambda_{n}^{-1 / 2}\right\|_{L^{2}(Q, \rho)}$ tend to 0 , see Theorems $2 \& 5$ of [11] or Theorems 3.5\&4.4 of [21]. In particular, if there is a unique solution $\boldsymbol{\mu}_{0}$ of Extremal Problem 1, then we get weak-* convergence of $\boldsymbol{\mu}_{n}$ to $\boldsymbol{\mu}_{0}$. A stronger result, involving narrow convergence of the total variation measure $\left|\boldsymbol{\mu}_{n}\right|$, can be found in Theorem 4.3 of [7]. This strengthening is important, for it implies that "no mass is lost" as could be the case with weak-* convergence, namely $\left\|\boldsymbol{\mu}_{n}\right\|_{T V}$ tends to $\left\|\boldsymbol{\mu}_{0}\right\|_{T V}$. To recap, we have a consistency property asserting that a magnetization meeting a certain assumptions (e.g. either (a), (b) or (c) above) can be approximately recovered via the regularization scheme (1.5), when the noise is small and the regularization parameter $\lambda$ is chosen small but still larger than the square of the noise (the so-called Morozov discrepancy principle). Note that (1.5) might a priori have several minimizers, for the objective function (1.4) is not strictly convex, as is easy to see.

In Section 3, we sharpen the analysis of [7] regarding Extremal Problems 1 and 2 in the case where $S$ is contained in a plane. We prove that $\boldsymbol{\mu}=\boldsymbol{\mu}_{0}$ is the unique solution to Extremal Problem 1 in case (c) listed above (Thm. 3.3), and also that Extremal Problem 2 has a unique solution for any data (Thm. 3.8). Both results depend on Theorem 2.8, asserting that a two-dimensional divergence-free measure $\boldsymbol{\nu}$ can be decomposed into loops; i.e., contour integrations along rectifiable Jordan curves such that $\mathrm{d} \boldsymbol{\nu} / \mathrm{d}|\boldsymbol{\nu}|(x)$ is, for $|\boldsymbol{\nu}|$-a.e. $x$, the unit tangent to any of these curves through $x$. This theorem is stated in Section 2.3, after some measure-theoretic preparation in Sections 2.1 and 2.2.

\subsection{Notation}

We conclude this section with some notation and definitions regarding measures and distributions. For a vector $x$ in the Euclidean space $\mathbb{R}^{n}$ (we mainly deal with $n=2$ or 3 ), we denote the $j$-th component of $x$ by $x_{j}$ and the partial derivative with respect to $x_{j}$ by $\partial_{x_{j}}$. By default, we consider vectors as column vectors; e.g., for $x \in \mathbb{R}^{3}$ we write $x=\left(x_{1}, x_{2}, x_{3}\right)^{T}$ where " $T$ " denotes "transpose". We write $\mathbb{N}$ for the nonnegative integers, $\mathbb{N}^{*}$ for the positive integers, and $\mathbb{R}^{+}$for the nonnegative real numbers. We use bold symbols to represent vector-valued functions and measures, and the corresponding nonbold symbols with subscripts to denote the respective components; e.g., $\boldsymbol{\mu}=\left(\mu_{1}, \mu_{2}, \mu_{3}\right)^{T}$ or $\mathbf{b}(\boldsymbol{\mu})=\left(b_{1}(\boldsymbol{\mu}), b_{2}(\boldsymbol{\mu}), b_{3}(\boldsymbol{\mu})\right)^{T}$. For $x \in \mathbb{R}^{n}$ and $R>0$, we let $\mathbb{B}(x, R)$ indicate the open ball centered at $x$ with radius $R$, and $\mathbb{S}(x, R)$ the boundary sphere. This notation does not show dependence on $n$, but no confusion will arise. We denote by $\mathcal{M}(E)$ the space of finite signed Borel measures on $E \subset \mathbb{R}^{n}$.

We write $\chi_{E}$ for the characteristic function of a set $E$ and $\delta_{x}$ for the Dirac delta measure at $x$. Given a $\mathbb{R}^{m}$-valued measure in $\boldsymbol{\mu} \in \mathcal{M}\left(\mathbb{R}^{n}\right)^{m}$ and a Borel set $E \subset \mathbb{R}^{n}$, we denote by $\boldsymbol{\mu}\lfloor E$ the measure obtained by restricting $\boldsymbol{\mu}$ to $E$ (i.e., for every Borel set $B \subset \mathbb{R}^{n}, \boldsymbol{\mu}\lfloor E(B):=\boldsymbol{\mu}(E \cap B)$ ).

For $\boldsymbol{\mu} \in \mathcal{M}\left(\mathbb{R}^{n}\right)^{m}$, the total variation measure $|\boldsymbol{\mu}|$ is defined on Borel sets $B \subset \mathbb{R}^{n}$ by

$$
|\boldsymbol{\mu}|(B):=\sup _{\mathcal{P}} \sum_{P \in \mathcal{P}}|\boldsymbol{\mu}(P)|,
$$

where the supremum is taken over all finite Borel partitions $\mathcal{P}$ of $B$. The total variation norm of $\boldsymbol{\mu}$ is then defined as

$$
\|\boldsymbol{\mu}\|_{T V}:=|\boldsymbol{\mu}|\left(\mathbb{R}^{n}\right) .
$$

The support of $\boldsymbol{\mu}$ is the complement of the largest open set $U$ such that $|\boldsymbol{\mu}|(U)=0$; it is denoted by $\operatorname{supp} \boldsymbol{\mu}$. A carrier for $\boldsymbol{\mu}$ is any $|\boldsymbol{\mu}|$-measurable set $E$ with full $|\boldsymbol{\mu}|$-measure; we also say that $\boldsymbol{\mu}$ is carried by $E$. Since $|\boldsymbol{\mu}|$ is a Radon measure, the Radon-Nikodym derivative $\mathbf{u}_{\boldsymbol{\mu}}:=\mathrm{d} \boldsymbol{\mu} / d|\boldsymbol{\mu}|$ exists as a $\mathbb{R}^{m}$-valued $|\boldsymbol{\mu}|$-integrable function and it satisfies $\left|\mathbf{u}_{\boldsymbol{\mu}}\right|=1$ a.e. with respect to $|\boldsymbol{\mu}|$. 
For $\Omega \subset \mathbb{R}^{n}$ an open set, we denote by $C_{c}\left(\Omega, \mathbb{R}^{m}\right)$ the space of $\mathbb{R}^{m}$-valued continuous functions with compact support on $\Omega$, equiped with the sup-norm. When $m=1$, we drop the dependence on $m$ and simply write $C_{c}(\Omega)$. A similar notational simplification is used for other functional spaces introduced below.

We shall identify $\boldsymbol{\mu} \in \mathcal{M}\left(\mathbb{R}^{n}\right)^{m}$ with the linear form on $C_{c}\left(\mathbb{R}^{n}, \mathbb{R}^{m}\right)$ given by

$$
\langle\boldsymbol{\mu}, \mathbf{f}\rangle:=\int \mathbf{f} \cdot \mathrm{d} \boldsymbol{\mu}, \quad \mathbf{f} \in C_{c}\left(\mathbb{R}^{n}, \mathbb{R}^{m}\right)
$$

The norm of the functional (1.8), is $\|\boldsymbol{\mu}\|_{T V}$. More generally, for $\Omega \subset \mathbb{R}^{n}$ an open set, it follows from Lusin's theorem ([26], Cor. to Thm. 2.23), applied to the restriction of $\mathbf{u}_{\boldsymbol{\mu}}$ to "large" compact sets in $\Omega$, and from the dominated convergence theorem that

$$
|\boldsymbol{\mu}|(\Omega)=\sup \left\{\langle\boldsymbol{\mu}, \boldsymbol{\varphi}\rangle, \boldsymbol{\varphi} \in C_{c}\left(\Omega, \mathbb{R}^{m}\right),|\boldsymbol{\varphi}| \leq 1\right\} .
$$

The functional (1.8) extends naturally with the same norm to the Banach space $C_{0}\left(\mathbb{R}^{n}, \mathbb{R}^{m}\right)$ of $\mathbb{R}^{m}$-valued continuous functions on $\mathbb{R}^{n}$ vanishing at infinity.

At places, we also identify $\boldsymbol{\mu}$ with the restriction of (1.8) to $C_{c}^{\infty}\left(\mathbb{R}^{n}, \mathbb{R}^{m}\right)$, the space of $C^{\infty}$-smooth functions with compact support, equiped with the usual topology of test functions [27]. We refer to a continuous linear functional on $C_{c}^{\infty}\left(\mathbb{R}^{n}, \mathbb{R}^{m}\right)$ as being a distribution, and put $\partial_{x_{i}}$ to mean distributional derivative with respect to the variable $x_{i}$.

We denote Lebesgue measure on $\mathbb{R}^{n}$ by $\mathcal{L}_{n}$ and $d$-dimensional Hausdorff measure by $\mathcal{H}^{d}$, see [15] for the definitions. We normalize $\mathcal{H}^{d}$ for $d=1$ and 2 so that it coincides with arclength and surface area for smooth curves and surfaces, and more generally that it agrees with $d$-dimensional volume for nice $d$-dimensional subsets of $\mathbb{R}^{n}$. We denote the Hausdorff dimension of a set $E$ by $\operatorname{dim}_{\mathcal{H}}(E)$. Recall that $E \subset R^{n}$ is $m$-rectifiable if it is the countable union of images of Lipschitz functions from $\mathbb{R}^{m}$ to $\mathbb{R}^{n}$, up to a set of $\mathcal{H}^{m}$-measure zero, see Definition 15.3 of [25].

For $E \subset \mathbb{R}^{n}$ a measurable set and $1 \leq p \leq \infty$, we write $L^{p}(E)$ for the familiar Lebesgue space of (equivalence classes of $\mathcal{L}_{n}$-a.e. coinciding) real-valued measurable functions on $E$ whose $p$-th power is integrable, with norm $\|g\|_{L^{p}(E)}=\left(\int_{E}|g|^{p} \mathrm{~d} \mathcal{L}_{n}\right)^{1 / p}$ (ess. $\sup _{E}|g|$ if $\left.p=\infty\right)$. If $E$ is open, we set $L_{l o c}^{1}(E)$ to consist of functions $f$ whose restriction $f_{\mid K}$ to $K$ lies in $L^{1}(K)$, for every compact $K \subset E$. For $\nu \in \mathcal{M}\left(\mathbb{R}^{n}\right)$ a positive measure different from $\mathcal{L}_{n}$, we put $L^{1}[\mathrm{~d} \nu]$ for the space of real-valued integrable functions against $\nu$.

We are particularly concerned with magnetizations supported on $\mathbb{R}^{2} \times\{0\} \subset \mathbb{R}^{3}$ and hence, with a slight abuse of notation, given $S \subset \mathbb{R}^{2}$ and $\boldsymbol{\mu} \in \mathcal{M}(S \times\{0\})^{3}$, we shall identify $S$ with $S \times\{0\} \subset \mathbb{R}^{3}$ and $\boldsymbol{\mu}$ with $\boldsymbol{\mu}\left\lfloor\left(\mathbb{R}^{2} \times\{0\}\right)\right.$. In addition, we let $\mathfrak{R}$ denote the rotation by $\pi / 2$ in $\mathbb{R}^{2}$; i.e., $\mathfrak{R}\left(\left(x_{1}, x_{2}\right)^{T}\right)=\left(-x_{2}, x_{1}\right)^{T}$.

For an open set $\Omega \subset \mathbb{R}^{n}$, recall the space $B V(\Omega)$ of functions of bounded variation comprised of functions in $L^{1}(\Omega)$ whose distributional derivatives are signed measures on $\Omega$ (see, [31]). We let $B V_{l o c}(\Omega)$ denote the space of functions whose restriction to any relatively compact open subset $\Omega_{1}$ of $\Omega$ lies in $B V\left(\Omega_{1}\right)$. We define the space $\dot{B V}(\Omega)$ of "homogeneous" BV-functions to consist of locally integrable functions whose distributional derivatives are finite signed measures on $\Omega$. Note that $\phi \in B \dot{B}(\Omega)$ if and only if it is a distribution on $\Omega$ such that $\nabla \phi \in \mathcal{M}(\Omega)^{n}$, by Theorem 6.7.7 of [14].

\section{MeAsure theoretical BaCKGround}

\subsection{Curves and divergence-free measures in $\mathbb{R}^{n}$}

For $a<b$ two real numbers, we call a Lipschitz mapping $\gamma:[a, b] \rightarrow \mathbb{R}^{n}$ a parametrized rectifiable curve, while the image $\Gamma:=\gamma([a, b])$ is simply termed a (non-parametrized) rectifiable curve. By Rademacher's Theorem (see $[15]), \gamma$ is differentiable a.e. on $[a, b]$. Note that $\gamma$ needs not be injective; i.e., the curve needs not be simple. If 
we let $N(\boldsymbol{\gamma}, x)$ be the cardinality (finite or infinite) of the preimage $\boldsymbol{\gamma}^{-1}(x)$, then the length $\ell(\gamma)$ of $\boldsymbol{\gamma}$ is

$$
\ell(\gamma):=\int_{a}^{b}\left|\gamma^{\prime}(t)\right| \mathrm{d} t=\int N(\boldsymbol{\gamma}, x) \mathrm{d} \mathcal{H}^{1}(x)
$$

where the second equality follows from the area formula ([18], 3.2.3). In particular, $\mathcal{H}^{1}(\Gamma)<\infty$ and $\mathcal{H}^{1}$-almost every $x \in \Gamma$ is attained only finitely many times by $\boldsymbol{\gamma}$. Observe that $\ell(\gamma) \neq \mathcal{H}^{1}(\Gamma)$ in general. When $\left|\boldsymbol{\gamma}^{\prime}(t)\right|=1$ a.e. on $[a, b]$, we call $\gamma$ a unit speed parametrization. This means that $\gamma$ parametrizes $\Gamma$ (non injectively perhaps) by percursed arclength.

If $\gamma$ is injective on $[a, b)$ and $\gamma(a)=\gamma(b)$, we say that $\gamma$ is a parametrized rectifiable Jordan curve and $\Gamma$ a rectifiable Jordan curve; in this case $\ell(\gamma)=\mathcal{H}^{1}(\Gamma)$. Given a Jordan curve $\Upsilon$ (i.e., the image of a circle by an injective continuous map) such that $\mathcal{H}^{1}(\Upsilon)<\infty$, one can easily construct a unit speed parametrization $\gamma:\left[0, \mathcal{H}^{1}(\Upsilon)\right] \rightarrow \Upsilon$ which is injective on $\left[0, \mathcal{H}^{1}(\Upsilon)\right)$ with $\gamma(0)=\gamma\left(\mathcal{H}^{1}(\Upsilon)\right)$. Thus, a Jordan curve $\Upsilon$ is rectifiable if and only if $\mathcal{H}^{1}(\Upsilon)<\infty$.

For $\gamma:[a, b] \rightarrow \mathbb{R}^{n}$ a parametrized rectifiable curve, we define $\mathbf{R}_{\gamma} \in \mathcal{M}\left(\mathbb{R}^{n}\right)^{n}$ by

$$
\left\langle\mathbf{R}_{\gamma}, \mathbf{g}\right\rangle:=\int_{a}^{b} \mathbf{g}(\gamma(t)) \cdot \gamma^{\prime}(t) \mathrm{d} t=\int_{\Gamma}\left(\sum_{t \in \gamma^{-1}(x)} \mathbf{g}(x) \cdot \gamma^{\prime}(t)\right) \mathrm{d} \mathcal{H}^{1}(x), \quad \mathbf{g} \in C_{0}\left(\mathbb{R}^{n}, \mathbb{R}^{n}\right),
$$

where the second equality follows from the area formula. Clearly, $\mathbf{R}_{\gamma}$ is supported on $\Gamma$ and $\left\|\mathbf{R}_{\gamma}\right\|_{T V} \leq \ell(\gamma)$. If we define $\psi:[a, b] \rightarrow[0, \ell(\gamma)]$ by $\psi(t)=\int_{a}^{t}\left|\gamma^{\prime}(\tau)\right| \mathrm{d} \tau$, then $\psi$ is Lipschitz with $\psi^{\prime}(t)=\left|\gamma^{\prime}(t)\right|$ a.e. and there is a unit speed parametrization $\widetilde{\gamma}:[0, \ell(\gamma)] \rightarrow \Gamma$ such that $\gamma=\widetilde{\gamma} \circ \psi$, by the chain rule and Sard's theorem for Lipschitz functions (see [25], Thm. 7.4). Moreover, we see from the area formula that $\mathbf{R}_{\boldsymbol{\gamma}}=\mathbf{R}_{\tilde{\gamma}}$, so we assume unless otherwise stated that parametrized rectifiable curves are unit speed parametrizations. When $\mathbf{g}$ is a gradient, say $\mathbf{g}=\nabla f$ for some $f \in C_{c}^{\infty}\left(\mathbb{R}^{n}\right)$, then $\left\langle\mathbf{R}_{\gamma}, \mathbf{g}\right\rangle=f(b)-f(a)$. Hence, $\mathbf{R}_{\boldsymbol{\gamma}}$ is divergence-free if and only if $\gamma$ is a closed curve.

One can check from (2.1) that $\mathbf{R}_{\gamma}$ is absolutely continuous with respect to $\mathcal{H}^{1}\lfloor\Gamma$ and has Radon-Nykodim derivative $d \mathbf{R}_{\gamma} / \mathrm{d}\left(\mathcal{H}^{1}\lfloor\Gamma)(x)=\sum_{t \in \boldsymbol{\gamma}^{-1}(x)} \gamma^{\prime}(t)\right.$ at $\mathcal{H}^{1}$-a.e. $x \in \boldsymbol{\Gamma}$, see Lemma A.1 of [6]. In particular, if $\gamma$ is a parametrized rectifiable Jordan curve then $\mathbf{R}_{\gamma}$ is absolutely continuous with respect to $\mathcal{H}^{1}\left\lfloor\Gamma\right.$, with $\gamma^{\prime} \circ \gamma^{-1}$ as Radon-Nikodym derivative.

It may happen that $\left\|\mathbf{R}_{\gamma}\right\|_{T V}<\ell(\boldsymbol{\gamma})$, because cancellation can occur in (2.1). For $\ell>0$, let $\mathcal{C}_{\ell}$ denote those $\mathbf{R}_{\gamma}$ such that $\left\|\mathbf{R}_{\gamma}\right\|_{T V}=\ell(\gamma)=\ell$. Then, $\mathbf{R}_{\gamma} \in \mathcal{C}_{\ell}$ if and only if $\Gamma$ has a well defined (oriented) unit tangent $\boldsymbol{\tau}(x)$ at $\mathcal{H}^{1}$-a.e. $x$, given by $\gamma^{\prime}(t)$ for any $t$ such that $\gamma(t)=x$, see Lemma A.2 of [6]. In particular, if $\gamma$ is a parametrized rectifiable Jordan curve of length $\ell$ then $\mathbf{R}_{\gamma} \in \mathcal{C}_{\ell}$.

It is shown in Theorem A of [28] that every $\boldsymbol{\mu} \in \mathcal{M}\left(\mathbb{R}^{n}\right)^{n}$ which is divergence-free is a superposition of members of $\mathcal{C}_{\ell}$, for fixed but arbitrary $\ell>0$. This means there is a finite positive Borel measure $\rho$ on the closed ball $\mathcal{B}_{\ell} \subset \mathcal{M}\left(\mathbb{R}^{n}\right)^{n}$ centered at 0 of radius $\ell$, which is carried by $\mathcal{C}_{\ell}$ and such that, for $\rho$-a.e. $\gamma$, the measure $\mathbf{R}_{\gamma}$ is supported in supp $\boldsymbol{\mu}$ and

$$
\langle\boldsymbol{\mu}, \mathbf{g}\rangle=\int_{\mathcal{C}_{\ell}}\left\langle\mathbf{R}_{\gamma}, \mathbf{g}\right\rangle \mathrm{d} \rho\left(\mathbf{R}_{\gamma}\right), \quad\langle|\boldsymbol{\mu}|, \varphi\rangle=\int_{\mathcal{C}_{\ell}}\left\langle\left|\mathbf{R}_{\gamma}\right|, \varphi\right\rangle \mathrm{d} \rho\left(\mathbf{R}_{\gamma}\right)
$$

for all $\mathbf{g} \in C_{c}^{\infty}\left(\mathbb{R}^{n}, \mathbb{R}^{n}\right)$ and $\varphi \in C_{c}^{\infty}\left(\mathbb{R}^{n}\right)$. Here, $\mathcal{M}\left(\mathbb{R}^{n}\right)^{n}$ is endowed with the weak-* topology that makes it a compact metrizable space, since its dual $C_{c}\left(\mathbb{R}^{n}, \mathbb{R}^{n}\right)$ is separable. Of course, by mollification, (2.2) holds more generally for $\mathbf{g} \in C_{c}\left(\mathbb{R}^{n}, \mathbb{R}^{n}\right)$ and $\varphi \in C_{c}\left(\mathbb{R}^{n}\right)$.

Decomposition (2.2) is highly non-unique (for one thing, $\ell>0$ is arbitrary), and the $\mathbf{R}_{\gamma}$ need not be divergence-free even though $\boldsymbol{\mu}$ is. Another decomposition of $\boldsymbol{\mu}$ as a superposition of so-called elementary solenoids is given in Theorem B of [28]; elementary solenoids are divergence-free, but their structure is more complex 
than the one of $\mathbf{R}_{\gamma}$. As asserted by Theorem 2.8 to come, a special situation prevails in the planar case $n=2$. Namely, if we let $\mathfrak{J} \subset \mathcal{M}\left(\mathbb{R}^{2}\right)^{2}$ be the set of normalized loops of the form $\ell(\gamma)^{-1} \mathbf{R}_{\gamma}$ where $\gamma:[a, b) \rightarrow \mathbb{R}^{2}$ is a Jordan curve, each divergence-free $\boldsymbol{\mu} \in \mathcal{M}\left(\mathbb{R}^{2}\right)^{2}$ is a superposition of members of $\mathfrak{J}$. This yields a decomposition of $\boldsymbol{\mu}$ into elementary solenoids which are loops.

Let us now recall a characterization of silent magnetizations with planar support. Since such a support is slender, the following lemma is a special case of Theorem 2.2 in [7].

Lemma 2.1. Let $S \subset \mathbb{R}^{2} \times\{0\}$ be closed and $\boldsymbol{\nu} \in \mathcal{M}(S)^{3}$. Then $\boldsymbol{\nu}$ is $S$-silent if and only if it is divergence-free; i.e., $\nabla \cdot \boldsymbol{\nu}=0$ in the distributional sense on $\mathbb{R}^{3}$.

The next elementary lemma, essentially contained in [5], characterizes the componentwise structure of silent planar magnetizations. We include a proof for the convenience of the reader. Recall the definition of $\dot{B V}$ and the notation $\mathfrak{R}$ for the rotation by $\pi / 2$ in $\mathbb{R}^{2}$.

Lemma 2.2. Let $S \subset \mathbb{R}^{2} \times\{0\}$ be closed, $\boldsymbol{\mu}=\left(\mu_{1}, \mu_{2}, \mu_{3}\right)^{T} \in \mathcal{M}(S)^{3}$, and $\boldsymbol{\mu}_{T}=\left(\mu_{1}, \mu_{2}\right)^{T}$. The following are equivalent:

1. $\nabla \cdot \boldsymbol{\mu}=0$ in the distributional sense on $\mathbb{R}^{3}$.

2. $\mu_{3}=0$ and $\nabla \cdot \boldsymbol{\mu}_{T}=0$ in the distributional sense on $\mathbb{R}^{2}$.

3. $\mu_{3}=0$ and $\boldsymbol{\mu}_{T}=\mathfrak{R} \nabla \phi=\left(-\partial_{x_{2}} \phi, \partial_{x_{1}} \phi\right)^{T}$ for some $\phi \in \dot{B V}\left(\mathbb{R}^{2}\right)$.

Proof. Since $\boldsymbol{\mu}$ has support contained in $\mathbb{R}^{2} \times\{0\}$, it can be written in tensor product form as $\boldsymbol{\mu}=\left(\boldsymbol{\mu}\left\lfloor\mathbb{R}^{2}\right) \otimes \delta_{x_{3}=0}\right.$ and thus $\nabla \cdot \boldsymbol{\mu}=\left(\nabla \cdot \boldsymbol{\mu}_{T}\right) \otimes \delta_{x_{3}=0}+\mu_{3} \otimes \delta_{x_{3}=0}^{\prime}$, where $\delta_{x_{3}=0}$ is the Dirac mass at zero on $\mathbb{R}$ in the variable $x_{3}$ and $\delta_{x_{3}=0}^{\prime}$ its distributional derivative. Hence, (b) implies that $\nabla \cdot \boldsymbol{\mu}=0$ and therefore (b)Rightarrow(a). Next, for any $\phi \in C_{c}^{\infty}\left(\mathbb{R}^{3}\right)$, let $\phi_{0}, \phi_{1} \in C_{c}^{\infty}\left(\mathbb{R}^{2}\right)$ be given by $\phi_{0}\left(x_{1}, x_{2}\right)=\phi\left(x_{1}, x_{2}, 0\right)$ and $\phi_{1}\left(x_{1}, x_{2}\right)=\partial_{x_{3}} \phi\left(x_{1}, x_{2}, 0\right)$. Then, it holds that

$$
\langle\nabla \cdot \boldsymbol{\mu}, \phi\rangle=-\left\langle\mu_{1}, \partial_{x_{1}} \phi_{0}\right\rangle-\left\langle\mu_{2}, \partial_{x_{2}} \phi_{0}\right\rangle-\left\langle\mu_{3}, \phi_{1}\right\rangle
$$

Pick $\phi$ of the form $\phi\left(x_{1}, x_{2}, x_{3}\right)=\psi\left(x_{1}, x_{2}\right) \eta\left(x_{3}\right)$ where $\psi \in C_{c}^{\infty}\left(\mathbb{R}^{2}\right)$ and $\eta \in C_{c}^{\infty}(\mathbb{R})$. First, letting $\eta$ be such that $\eta(0)=1$ and $\eta^{\prime}(0)=0$, we deduce from (2.3) that if $\nabla \cdot \boldsymbol{\mu}=0$ then $\nabla \cdot \boldsymbol{\mu}_{T}=0$. Second, letting $\eta$ be such that $\eta(0)=0$ and $\eta^{\prime}(0)=1$, we deduce from (2.3) again that if $\nabla \cdot \boldsymbol{\mu}=0$ then $\mu_{3}=0$, whence (a) $\mathbb{R}$ ightarrow(b).

Suppose now that (b) holds. Then $\left(-\mu_{2}, \mu_{1}\right)^{T}$ satisfies the Schwartz rule when viewed as a $\mathbb{R}^{2}$ valued distribution on $\mathbb{R}^{2}$; i.e, $\partial_{x_{2}}\left(-\mu_{2}\right)=\partial_{x_{1}} \mu_{1}$. Therefore, $\mathfrak{R} \boldsymbol{\mu}_{T}=\left(-\mu_{2}, \mu_{1}\right)^{T}$ is the gradient of a scalar valued distribution $\Psi$ (see, [27]). Since the components of $\nabla \Psi$ are finite signed measures, $\Psi \in B V_{\text {loc }}$ ([14], Thm. 6.7.7) so that in fact $\Psi \in B V\left(\mathbb{R}^{2}\right)$. Thus, (c) holds with $\phi=-\Psi$ and we get that (b) Rightarrow(c). In the other direction if $\boldsymbol{\mu}_{T}=\left(-\partial_{x_{2}} \phi, \partial_{x_{1}} \phi\right)^{T}$ for some distribution $\phi$, then $\nabla \cdot \boldsymbol{\mu}_{T}=-\partial_{x_{1}} \partial_{x_{2}} \phi+\partial_{x_{2}} \partial_{x_{1}} \phi=0$ so that (c) Rightarrow(b).

Lemma 2.2 entails that decomposing solenoids in the plane as a superposition of loops is equivalent, up to a rotation, to decomposing gradients as a superposition of unit normal fields to a family of Jordan curves. As proposed in [28], this can be achieved via the co-area formula for $B V$-functions and the decomposition of 1-dimensional integral currents as a sum of indecomposable elements ([18], 4.2.25). A specific instance of the latter, used in the present context, is the decomposition into Jordan curves of the measure-theoretic boundary of planar sets of finite perimeter given in [1]. We discuss the necessary material in the forthcoming section.

\subsection{Sup-level sets of functions in $\dot{B V}\left(\mathbb{R}^{n}\right)$ and the co-area formula}

For $E \subset \mathbb{R}^{n}$ a Borel set, the measure-theoretical boundary of $E$ is the set $\partial_{M} E$ defined by

$$
\partial_{M} E:=\left\{x \in \mathbb{R}^{n}: \limsup _{\rho \rightarrow 0} \frac{\mathcal{L}_{n}(\mathbb{B}(x, \rho) \cap E)}{\mathcal{L}_{n}(\mathbb{B}(x, \rho))}>0 \text { and } \limsup _{\rho \rightarrow 0} \frac{\mathcal{L}_{n}(\mathbb{B}(x, \rho) \backslash E)}{\mathcal{L}_{n}(\mathbb{B}(x, \rho))}>0\right\} .
$$


Note that $\partial_{M} E$ is a subset of the topological boundary of $E$.

A measurable set $E \subset \mathbb{R}^{n}$ such that $\nabla \chi_{E} \in \mathcal{M}\left(\mathbb{R}^{n}\right)^{n}$ is said to be of finite perimeter ${ }^{1}$. For such a set it holds that

$$
\left|\nabla \chi_{E}\right|=\mathcal{H}^{n-1}\left\lfloor\partial_{M} E\right.
$$

and $\left\|\nabla \chi_{E}\right\|_{T V}=\mathcal{H}^{n-1}\left(\partial_{M} E\right)$ is called the perimeter of $E$. The identity (2.5) can be obtained by combining ([15], Thm. 5.15 (iii)), saying that (2.5) holds when $\partial_{M} E$ is replaced by the so-called reduced boundary of $E$, with Lemma 5.5 of [15], asserting that $\partial_{M} E$ differs from the reduced boundary by a set of $\mathcal{H}^{n-1}$-measure zero (see also [4], Thm. 10.3.2).

It follows from (2.5) that a set of finite perimeter has a measure-theoretical boundary of finite $\mathcal{H}^{n-1}$-measure. In contrast, its Euclidean boundary can be much larger and even have positive $\mathcal{L}_{n}$-measure, as the following example shows.

Example 2.3. Let $E_{1}=\overline{\mathbb{B}}(0,1) \subset \mathbb{R}^{2}$ and $\left\{q_{j}\right\}_{j \in \mathbb{N}}$ enumerate all points in $E_{1}$ with rational coordinates. Having defined inductively a closed set $E_{n}$ for $n \geq 1$, let $j_{n}$ be the smallest integer such that $q_{j_{n}}$ lies interior to $E_{n}$ and $B_{n}$ the largest open ball centered at $q_{j_{n}}$ contained in $E_{n}$, with radius $r_{n} \leq 2^{-n}$ ( at some steps $B_{n}$ could be empty). Then, define $E_{n+1}=E_{n} \backslash B_{n}$ which must be a closed set with nonempty interior, otherwise a finite union of balls of total $\mathcal{L}_{2}$-measure less than $\pi / 3$ would cover $\mathbb{B}(0,1)$. Hence, the process can continue indefinitely, and we let $E=\bigcap E_{n}$ which is a closed set.

Clearly $E$ has no interior, for all the $q_{j}$ have been excised out in the process; therefore its Euclidean boundary is $E$ itself. Moreover, $\mathcal{L}_{2}(E) \geq \pi-\pi \sum_{n=1}^{\infty} r_{n}^{2} \geq \pi\left(1-\sum_{n=1}^{\infty} 4^{-n}\right)>0$.

Now, by the standard Green formula, each $E_{n}$ is of finite perimeter, because it is a finitely connected set with piecewise smooth boundary. Thus, $\left\{\chi_{E_{n}}\right\}$ is a nonincreasing sequence of $B V$-functions and their point-wise limit $\chi_{E}$ is integrable. Also, by (2.5), it holds that $\left\|\nabla \chi_{E_{n}}\right\|_{T V} \leq 2 \pi \sum_{n=0}^{\infty} r_{n} \leq 4 \pi$, therefore we can use ([31], Rem. 5.2.2) to the effect that $\chi_{E} \in B V\left(\mathbb{R}^{2}\right)$; i.e. $E$ is a set of finite perimeter with Euclidean boundary of positive $\mathcal{L}_{2}$-measure, as announced.

For any $E \subset \mathbb{R}^{n}$ of finite perimeter, one defines the generalized unit inner normal $\boldsymbol{\nu}_{E}$ to $\partial_{M} E$ as the RadonNikodym derivative $\mathbf{u}_{\nabla \chi_{E}}$ which is but $d \nabla \chi_{E} / \mathrm{d}\left(\mathcal{H}^{n-1}\left\lfloor\partial_{M} E\right)\right.$, by (2.5). Then, the Radon Nikodym Theorem entails the following version of the Gauss-Green formula:

if $E \subset \mathbb{R}^{n}$ is a set of finite perimeter, then for each Borel set $B \subset \mathbb{R}^{n}$ it holds that

$$
\nabla \chi_{E}(B)=\int_{B} \boldsymbol{\nu}_{E} \mathrm{~d}\left(\mathcal{H}^{n-1}\left\lfloor\partial_{M} E\right)\right.
$$

The connection with the classical Gauss-Green formula is more transparent on the distributional version of (2.6), namely:

$$
\int \chi_{E} \nabla \cdot \boldsymbol{\varphi} \mathrm{d} \mathcal{L}_{n}=-\int \boldsymbol{\varphi} \cdot \boldsymbol{\nu}_{E} \mathrm{~d}\left(\mathcal{H}^{n-1}\left\lfloor\partial_{M} E\right), \quad \boldsymbol{\varphi} \in C_{c}^{1}\left(\mathbb{R}^{n}, \mathbb{R}^{n}\right)\right.
$$

The identity $(2.7)$ was proven in $[12,13,16,17]$; see also Theorem 5.16 of [15] and Theorem 10.3 .2 of [4]. Note that if $E$ has finite perimeter, then so does $\mathbb{R}^{n} \backslash E$ and $\boldsymbol{\nu}_{\mathbb{R}^{n} \backslash E}=-\boldsymbol{\nu}_{E}$.

Remark 2.4. When $n=2$, we see from (2.7) that $\boldsymbol{\nu}_{E}$ coincides with the usual, differential-geometric inner unit normal to the boundary of $E$ when the latter is the interior of a rectifiable Jordan curve, for in this case the Gauss-Green formula is valid for both definitions of the normal (see ([3], Thm. 10-43) for a suitable version of the Gauss-Green formula). Actually, the measure-theoretical boundary of a planar set of finite perimeter is

\footnotetext{
${ }^{1}$ In $[4,15,31]$, the definition is that $\chi_{E} \in B V\left(\mathbb{R}^{n}\right)$. The present definition means that $\chi_{E} \in B \dot{B}\left(\mathbb{R}^{n}\right)$ and, in view of Theorem 3.47 in [2], amounts to requiring that either $\chi_{E}$ or $\chi_{\mathbb{R}^{n} \backslash E}$ lies in $B V\left(\mathbb{R}^{n}\right)$.
} 
comprised of a countable union of rectifiable Jordan curves, up to a set of $\mathcal{H}_{1}$-measure zero ([1], Cor. 1). Thus, both notions of inner unit normal coincide $\mathcal{H}^{1}$-a.e. on the measure-theoretical boundary of such a set.

Given a function $\phi \in L_{l o c}^{1}\left(\mathbb{R}^{n}\right)$, we consistently denote with $E_{t}$ the suplevel sets:

$$
E_{t}:=\left\{x \in \mathbb{R}^{n} \mid \phi(x)>t\right\}
$$

Of course, the set $E_{t}$, as well as a number of subsequent sets in $\mathbb{R}^{n}$ that we will consider, is defined up to a set of $\mathcal{L}_{n}$-measure zero only, but which representative is chosen will be irrelevant for our purposes. Hereafter, we abbreviate the sentence "up to a set of $\mathcal{L}_{n}$-measure zero" by "mod- $\mathcal{L}_{n}$ ", and similarly for $\mathcal{H}^{n-1}$.

The sup-level sets are instrumental in the co-area (or Fleming-Rishel) formula for $\dot{B} V$-functions, of which we record a version in Lemma 2.5 below; see, e.g. Theorem 3.40 of [2].

Lemma 2.5. Suppose $\phi \in \dot{B V}\left(\mathbb{R}^{n}\right)$ and let $E_{t}$ be as in (2.8). Then, $E_{t}$ has finite perimeter for a.e. $t \in \mathbb{R}$ and for any Borel set $B \subset \mathbb{R}^{n}, \mathbf{g} \in L^{1}[\mathrm{~d}|\nabla \phi|]^{n}$ and $h \in L^{1}[\mathrm{~d}|\nabla \phi|]$, it holds that

$$
\begin{aligned}
& \text { 1. }|\nabla \phi|(B)=\int_{-\infty}^{\infty}\left|\nabla \chi_{E_{t}}\right|(B) \mathrm{d} t=\int_{-\infty}^{\infty} \mathcal{H}^{n-1}\left(\partial_{M} E_{t} \cap B\right) \mathrm{d} t, \\
& \text { 2. } \int h \mathrm{~d}(|\nabla \phi|)=\int_{-\infty}^{\infty} \int h \mathrm{~d}\left(\left|\nabla \chi_{E_{t}}\right|\right) \mathrm{d} t=\int_{-\infty}^{\infty} \int h \mathrm{~d}\left(\mathcal{H}^{n-1}\left\lfloor\partial_{M} E_{t}\right) \mathrm{d} t,\right. \\
& \text { 3. } \nabla \phi(B)=\int_{-\infty}^{\infty} \nabla \chi_{E_{t}}(B) \mathrm{d} t=\int_{-\infty}^{\infty} \int_{B} \boldsymbol{\nu}_{E_{t}} \mathrm{~d}\left(\mathcal{H}^{n-1}\left\lfloor\partial_{M} E_{t}\right) \mathrm{d} t,\right. \\
& \text { 4. } \int \mathbf{g} \cdot \mathrm{d}(\nabla \phi)=\int_{-\infty}^{\infty} \int \mathbf{g} \cdot \mathrm{d}\left(\nabla \chi_{E_{t}}\right) \mathrm{d} t=\int_{-\infty}^{\infty} \int \mathbf{g} \cdot \boldsymbol{\nu}_{E_{t}} \mathrm{~d}\left(\mathcal{H}^{n-1}\left\lfloor\partial_{M} E_{t}\right) \mathrm{d} t,\right.
\end{aligned}
$$

where in (b) the function $h$ lies in both $L^{1}\left[\mathrm{~d}\left|\nabla \chi_{E_{t}}\right|\right]$ and $L^{1}\left[\mathrm{~d} \mathcal{H}^{n-1}\left\lfloor\partial_{M} E_{t}\right]\right.$ for a.e. $t$ and in (d) the functions $\mathbf{g}$ and $\mathbf{g} \cdot \boldsymbol{\nu}_{E_{t}}$ lie in $L^{1}\left[\mathrm{~d}\left|\nabla \chi_{E_{t}}\right|\right]^{n}$ and $L^{1}\left[\mathrm{~d} \mathcal{H}^{n-1}\left\lfloor\partial_{M} E_{t}\right]\right.$, respectively, for a.e. $t$.

In fact, that $E_{t}$ has finite perimeter for a.e. $t$ and that (a) and (c) hold follows from Theorem 3.40 of [2] and (2.6). Then, (a) and (c) respectively yield (b) and (d) for simple functions, and the general case follows by dominated convergence, using (a) to ascertain that a Borel set $B$ such that $|\nabla \phi|(B)=0$ has $\left|\nabla \chi_{E_{t}}\right|(B)=0$ and $\mathcal{H}^{n-1}\left\lfloor\partial_{M} E_{t}(B)=0\right.$ for a.e. $t$.

One can also describe the "measure theoretical discontinuities" of $\dot{B} V$-functions as follows. For $\Omega \subset \mathbb{R}^{n}$ an open set and a $\mathcal{L}_{n}$-measurable $f: \Omega \rightarrow \mathbb{R}$, define for $x \in \mathbb{R}^{n}$ (see [15], Def. 5.8, 5.9):

$$
\begin{gathered}
f^{\text {sup }}(x):=\operatorname{ap} \limsup _{y \rightarrow x} f(y)=\inf \left\{t \mid \lim _{r \rightarrow 0} \frac{\mathcal{L}_{n}(\mathbb{B}(x, r) \cap\{\phi>t\})}{\mathcal{L}_{n}(\mathbb{B}(x, r))}=0\right\}, \\
f^{\text {inf }}(x):=\operatorname{ap} \liminf _{y \rightarrow x} f(y)=\sup \left\{t \mid \lim _{r \rightarrow 0} \frac{\mathcal{L}_{n}(\mathbb{B}(x, r) \cap\{\phi<t\})}{\mathcal{L}_{n}(\mathbb{B}(x, r))}=0\right\} \\
\text { and } J(f):=\left\{x \mid f^{\text {inf }}(x)<f^{\sup }(x)\right\} .
\end{gathered}
$$

Lemma 2.6. Given $\phi \in \dot{B} V\left(\mathbb{R}^{n}\right)$, the set $J(\phi)$ is $(n-1)$-rectifiable. Furthermore, $\nabla \phi\lfloor J(\phi)$ is absolutely continuous with respect to $\mathcal{H}^{n-1}$ and, with $E_{t}$ as in (2.8), its Radon-Nykodim derivative satisfies for a.e. $t \in \mathbb{R}$ and $\mathcal{H}^{n-1}$-a.e. $x \in \partial_{M} E_{t} \cap J: d \nabla \phi / \mathrm{d} \mathcal{H}^{n-1}=\left(\phi^{\text {sup }}-\phi^{\text {inf }}\right) \boldsymbol{\nu}_{E_{t}}$.

Proof. Clearly, it is enough that the result holds for the restriction $\phi_{\mid B}$ of $\phi$ to a bounded open ball $B \subset \mathbb{R}^{n}$, with $E_{t}$ replaced by $E_{t} \cap B$ and $J(\phi)$ by $J(\phi) \cap B$. Since $\phi_{\mid B} \in B V(B)$, the conclusion now follows from Remark 10.3.4, Theorem 10.4.1 of [4], see also Theorem 3.78 of [2]. 


\subsection{Loop decomposition of divergence-free planar measures}

Let us make one more piece of notation: for $\Gamma \subset \mathbb{R}^{2}$ a Jordan curve, we denote by int $(\Gamma)$ the bounded connected component of $\mathbb{R}^{2} \backslash \Gamma$ and by by $\operatorname{ext}(\Gamma)$ its bounded connected component. We record for later use the following "obvious" lemma.

Lemma 2.7. If $\Gamma \subset \mathbb{R}^{2}$ is a rectifiable Jordan curve, then $\partial_{M}(\operatorname{int}(\Gamma))=\partial_{M}(\operatorname{ext}(\Gamma))=\Gamma$ up to a set of $\mathcal{H}^{1}$ measure zero.

Proof. Clearly $\partial_{M}(\operatorname{int}(\Gamma))=\partial_{M}(\operatorname{ext}(\Gamma)) \subset \Gamma$. By Proposition $2 \&$ Theorem 7 of $[1], \partial_{M}(\operatorname{int}(\Gamma))$ is a rectifiable Jordan curve $\tilde{\Gamma}$, up to a set of $\mathcal{H}^{1}$-measure zero. Thus, $\mathcal{H}^{1}(\tilde{\Gamma} \backslash \Gamma)=0$ whence $\tilde{\Gamma} \cap \Gamma$ is dense in $\tilde{\Gamma}$, and so $\tilde{\Gamma} \subset \Gamma$ by compactness of $\Gamma$. Therefore, by the Jordan curve theorem, $\tilde{\Gamma}=\Gamma$.

We regard a parametrized Jordan curve $\boldsymbol{\gamma}$ as the member $\mathbf{R}_{\boldsymbol{\gamma}}$ of $\mathcal{M}\left(\mathbb{R}^{2}\right)^{2}$ defined in (2.1). Here, a degenerate curve has constant parametrization and therefore corresponds to the zero measure. We endow parametrized Jordan curves with the weak-* topology inherited from $\mathbb{R}^{2}$-valued measures.

Let $\mathcal{C}$ denote the space of sequences $\left\{\gamma_{n}\right\}_{n \in \mathbb{N}}$ of parametrized Jordan curves in $\mathbb{R}^{2}$ whose lengths are summable: $\sum_{n \in \mathbb{N}} \ell\left(\gamma_{n}\right)<\infty$. We let $\dot{\mathcal{C}}$ indicate the set of equivalence classes under permutation of the terms in the sequence. Recalling that $\mathcal{M}\left(\mathbb{R}^{2}\right)^{2}$ equipped with the weak-* topology is a metric space, say with distance $d_{w}$, we endow $\mathcal{C}$ with the distance $d_{\mathcal{C}}\left(\left(\gamma_{n}\right),\left(\boldsymbol{\gamma}_{n}^{\prime}\right)\right):=\sup _{n} d_{w}\left(\mathbf{R}_{\gamma_{n}}, \mathbf{R}_{\gamma_{n}^{\prime}}\right)$ and $\dot{\mathcal{C}}$ with the quotient topology.

Recall that a map $\psi: \mathbb{R} \rightarrow \mathcal{E}$, with $\mathcal{E}$ a topological space, is approximately continuous at $t_{0} \in \mathbb{R}$ if, for every neighborhood $V \subset \mathcal{E}$ of $\psi\left(t_{0}\right)$, it holds that

$$
\lim _{r \rightarrow 0} \frac{\mathcal{L}_{1}\left(\left\{t:\left|t-t_{0}\right|<r, \psi(t) \notin V\right\}\right)}{r}=0 .
$$

We can now state a representation theorem for divergence-free measures in the plane as a superposition of loops. This is Theorem 2.8 below, in which we point out that the second assertion of item (iii) is not proven. Indeed, the argument is somewhat heavy and the corresponding assertion will not be used later on. A full proof can be found in Theorem 4.5 of [6], see discussion after Theorem 2.8.

Theorem 2.8. Let $\boldsymbol{\nu} \in \mathcal{M}(S)^{2}$. Then $\boldsymbol{\nu}$ is divergence-free in $\mathbb{R}^{2}$ if and only if there exists $G \subset \mathbb{R}$ with $\mathcal{L}_{1}(\mathbb{R} \backslash$ $G)=0$ such that, for each $t \in G$, there is a countable collection of (possibly degenerate) parametrized rectifiable Jordan curves $\left\{\gamma_{n}^{t}\right\}_{n \in \mathbb{N}}$ with images $\Gamma_{n}^{t}$ such that:

(i) the $\left\{\Gamma_{n}^{t}\right\}_{n \in \mathbb{N}}$ are disjoint up to a set of $\mathcal{H}^{1}$-measure zero and $\Gamma_{n}^{t} \subset \operatorname{supp} \boldsymbol{\nu}$ for each $n$;

(ii) the union $\bigcup_{n} \Gamma_{n}^{t}$ is, up to a set of $\mathcal{H}^{1}$-measure zero, the measure-theoretical boundary $\partial_{M} \Omega(t)$ of a set $\Omega(t) \subset \mathbb{R}^{2}$ of finite perimeter;

(iii) $\Omega\left(t_{1}\right) \supset \Omega\left(t_{2}\right)$ if $t_{1}<t_{2}$, and the mapping $t \mapsto\left\{\gamma_{n}^{t}\right\}_{n \in \mathbb{N}}$ from $\mathbb{R}$ to $\dot{\mathcal{C}}$ is approximately continuous at a.e. $t$;

(iv) For any Borel set $B \subset \mathbb{R}^{2}, \mathbf{g} \in L^{1}[\mathrm{~d}|\boldsymbol{\nu}|]^{2}$ and $h \in L^{1}[\mathrm{~d}|\boldsymbol{\nu}|]$, it holds that

$$
\boldsymbol{\nu}(B)=\int_{\mathbb{R}} \sum_{n \in \mathbb{N}}\left(\int_{B} \boldsymbol{\tau}_{n}^{t} \mathrm{~d}\left(\mathcal{H}^{1}\left\lfloor\Gamma_{n}^{t}\right)\right) \mathrm{d} t,\right.
$$

where $\boldsymbol{\tau}_{n}^{t}=\left(\gamma_{n}^{t}\right)^{\prime} /\left|\left(\gamma_{n}^{t}\right)^{\prime}\right|$ is the unit tangent vector field to $\Gamma_{n}^{t}$ oriented by $\boldsymbol{\gamma}_{n}^{t}$,

$$
|\boldsymbol{\nu}|(B)=\int_{\mathbb{R}} \mathcal{H}^{1}\left(\partial_{M} \Omega(t) \cap B\right) \mathrm{d} t=\int_{\mathbb{R}}\left(\sum_{n \in \mathbb{N}} \mathcal{H}^{1}\left(\Gamma_{n}^{t} \cap B\right)\right) \mathrm{d} t
$$




$$
\int \mathbf{g} \cdot \mathrm{d} \boldsymbol{\nu}=\int_{\mathbb{R}} \sum_{n \in \mathbb{N}}\left(\int \mathbf{g} \cdot \boldsymbol{\tau}_{n}^{t} \mathrm{~d}\left(\mathcal{H}^{1}\left\lfloor\Gamma_{n}^{t}\right)\right) \mathrm{d} t\right.
$$

and

$$
\int h \mathrm{~d}|\boldsymbol{\nu}|=\int_{\mathbb{R}} \sum_{n \in \mathbb{N}}\left(\int h \mathrm{~d}\left(\mathcal{H}^{1}\left\lfloor\Gamma_{n}^{t}\right)\right) \mathrm{d} t\right.
$$

where the inner integrals on the right handsides of (2.13) and (2.14) are well defined for a.e. $t \in \mathbb{R}$.

(v) The set $J:=\bigcup_{\substack{t_{1} \neq t_{2} \in G \\ n_{1}, n_{2} \in \mathbb{N}}}^{t_{n_{1}}} \cap \Gamma_{n_{2}}^{t_{2}}$ is 1-rectifiable in $\mathbb{R}^{2}$ and $\boldsymbol{\nu}\left\lfloor J\right.$ is absolutely continuous with respect to $\mathcal{H}^{1}$; for a.e. $t \in G, \mathbf{u}_{\boldsymbol{\nu}}(x)=\boldsymbol{\tau}_{n}^{t}(x)$ for $\mathcal{H}^{1}$-a.e. $x \in J \cap \partial_{M} \Omega(t)$. More generally, it holds for a.e. $t \in G$ and every $n \in \mathbb{N}$ that $\mathbf{u}_{\boldsymbol{\nu}}(x)=\boldsymbol{\tau}_{n}^{t}(x)$ for $\mathcal{H}^{1}$-a.e. $x \in \Gamma_{n}^{t}$.

Proof. Any $\boldsymbol{\nu}$ of the form (2.11) is divergence-free (see [28] or discussion following (2.1)).

Now suppose $\boldsymbol{\nu}$ is divergence-free. By Lemma 2.2, we have $\boldsymbol{\nu}(B)=\mathfrak{R} \nabla \phi(B)$ for some $\phi \in \dot{B V}\left(\mathbb{R}^{2}\right)$. Defining $E_{t}$ as in (2.8), we get from Lemma 2.5 that it has finite perimeter for a.e. $t$. Let $G$ be the set of such $t$, and for $t \in G$ write $\left\{\gamma_{n}^{t}\right\}_{n \in \mathbb{N}}$ for the family of parametrized Jordan curves constitutive of $\partial_{M} E_{t}$ ([1], Cor. 1), augmented by infinitely many degenerate curves reducing to a point in case this family is finite. Here, we orient $\gamma_{n}^{t}$ so that $\boldsymbol{\tau}_{n}^{t}=\Re \nu_{E_{t}}$ at $\mathcal{H}^{1}$-a.e. point of $\boldsymbol{\gamma}_{n}^{t}$. The ordering $n \mapsto \boldsymbol{\gamma}_{n}^{t}$ is arbitrary, but when taking the equivalence class in $\dot{\mathcal{C}}$ of $\left\{\gamma_{n}^{t}\right\}_{n \in \mathbb{N}} \in \mathcal{C}$, this ordering becomes immaterial. Now, if we set $\Omega(t)=E_{t}$, then (ii) and the first assertion in (i) come from Corollary 1 of [1] and the first assertion in (iii) is obvious; as pointed out before the theorem, we refer the reader to Theorem 4.5 of [6] for the proof of the second assertion on approximate continuity. Recalling definition (2.2), we see that Lemma 2.5 and the remark after (2.7) together imply $(i v)$, where it should be noted that equations (2.11) through (2.14) only depend on the equivalence class of $\left\{\gamma_{n}^{t}\right\}_{n \in \mathbb{N}}$ in $\dot{\mathcal{C}}$. Since (2.12) implies that $\mathcal{H}^{1}\left(\Gamma_{n}(t) \backslash \operatorname{supp} \boldsymbol{\nu}\right)=0$ for a.e. $t \in \mathbb{R}$ the second half of $(i)$ holds.

Observing that $\bigcup_{n \in \mathbb{N}} \Gamma_{n}^{t}=\partial_{M} E_{t} \bmod -\mathcal{H}^{1}$, we see for each $t \in G$ that every $x \in J$ lies in $\partial_{M}\left(\mathbb{R}^{2} \backslash E_{t_{1}}\right) \cap \partial_{M} E_{t_{2}}$ for some $t_{1}<t_{2}$. Remembering the definitions in (2.9), this implies that, for every $x \in J, \phi^{\text {inf }}(x) \leq t_{1}<t_{2} \leq$ $\phi^{\text {sup }}(x)$. Hence, by Lemma 2.6, $J \subset J(\phi)$ and the first two assertions of $(v)$ follow. Now, evaluating $\|\boldsymbol{\nu}\|$ with (2.12) and integrating (2.13) against $\mathbf{u}_{\boldsymbol{\nu}}$ we get,

$$
\int_{\mathbb{R}}\left(\sum_{n \in \mathbb{N}} \mathcal{H}^{1}\left(\Gamma_{n}^{t}\right)\right) \mathrm{d} t=\|\boldsymbol{\nu}\|=\int \mathbf{u}_{\boldsymbol{\nu}} \cdot \mathrm{d} \boldsymbol{\nu}=\int_{\mathbb{R}} \sum_{n \in \mathbb{N}}\left(\int \mathbf{u}_{\boldsymbol{\nu}} \cdot \boldsymbol{\tau}_{n}^{t} \mathrm{~d}\left(\mathcal{H}^{1}\left\lfloor\Gamma_{n}^{t}\right)\right) \mathrm{d} t,\right.
$$

and noting that $\mathbf{u}_{\nu} \cdot \boldsymbol{\tau}_{n}^{t} \leq 1$, with equality only when $\mathbf{u}_{\nu}=\boldsymbol{\tau}_{n}^{t}$, gives us the last assertion of $(v)$.

The unproven assertion in (iii) of Theorem 2.8, claiming approximate continuity of $t \mapsto\left\{\gamma_{n}^{t}\right\}_{n \in \mathbb{N}}$ from $\mathbb{R}$ to $\dot{\mathcal{C}}$ at a.e. $t$, is key to establish that $\rho:=\mathrm{d} t \otimes \sum_{n} \mathcal{H}^{1}\left(\Gamma_{n}^{t}\right) \delta_{\ell^{-1}\left(\gamma_{n}^{t}\right) \mathbf{R}_{\gamma_{n}^{t}}}$ is a Borel measure on the unit ball of $\mathcal{M}\left(\mathbb{R}^{2}\right)^{2}$ which is carried by $\mathfrak{J}$ (cf. definition after (2.2)) and satisfies $\boldsymbol{\mu}=\int_{J \in \mathfrak{J}} J \mathrm{~d} \rho(J)$ as well as $|\boldsymbol{\mu}|=\int_{J \in \mathfrak{J}}|J| \mathrm{d} \rho(J)$. This gives a fairly concrete decomposition of a divergence-free measure as a superposition of elementary solenoids in the planar case, and we refer the reader to Proposition 4.6 of [6] for a proof. Let us also mention that, even though $\partial_{M} \Omega(t)$ is approximately continuous with respect to $t$ in the weak-* sense by what precedes, the $\Omega(t)$ in Theorem 2.8 may all have different topologies, as can be seen from the following example.

Example 2.9. We will generate a $B V$ function $\varphi_{\infty}$, valued in $[0,1]$, whose suplevel sets $E_{t}$ all have different topologies. Then, $\boldsymbol{\nu}:=\mathfrak{R} \nabla \varphi_{\infty}$ is divergence-free and $\Omega(t)=E_{t}$ in Theorem 2.8, thereby yielding an example with the aforementioned property.

We construct $\varphi_{\infty}$ as the limit of a bounded increasing sequence $\left(\phi_{m}\right)$ of $B V$ functions. Let us first define a family of sets of finite perimeter that we will use to construct the $\phi_{m}$. For any two integers $m$ and $n$ such that $m \geq 0$ and $1 \leq n \leq 2^{m}$, define the set $b(n, m) \subset \mathbb{R}^{2}$ to be the closed ball around the point $(n, m)$ with 
perimeter $2^{-2 m-1}$ (thus, radius $2^{-2 m-2} / \pi$ ) minus $2^{m}$ pairwise disjoint nonempty open balls contained in this closed ball. We pick the sum of the perimeters of this $2^{m}$ open balls to be strictly less than $2^{-2 m-1}$. Note that the $b(n, m)$ are pairwise disjoint. Define $\varphi_{0}:=\frac{1}{2} \chi_{b(1,0)}$ and, for $m>0, \varphi_{m}:=\varphi_{m-1}+\sum_{k=1}^{2^{m}} \frac{2 k-1}{2^{m+1}} \chi_{b(k, m)}$. Then $\left\|\nabla \varphi_{0}\right\|_{T V}<1 / 2$, moreover for $m>0$ :

$$
\begin{aligned}
\left\|\nabla \varphi_{m}\right\|_{T V} & =\left\|\nabla \varphi_{m-1}\right\|_{T V}+\sum_{k=1}^{2^{m}} \frac{2 k-1}{2^{m+1}}\left\|\nabla \chi_{b(k, m)}\right\|_{T V} \\
& <\left\|\nabla \varphi_{m-1}\right\|_{T V}+\sum_{k=1}^{2^{m}} \frac{2 k-1}{2^{m+1}}\left(2^{-2 m-1}+2^{-2 m-1}\right) \\
& =\left\|\nabla \varphi_{m-1}\right\|_{T V}+\frac{2^{2 m}}{2^{3 m+1}},
\end{aligned}
$$

and hence, $\left\|\nabla \varphi_{m}\right\|_{T V}<1$ for every $m$. Thus, $\varphi_{\infty}$, the pointwise limit of the nondecreasing sequence of functions $\left\{\varphi_{m}\right\}_{m}$, is a BV function (see [31], Thm. 5.2.1).

Now, for $m, n, p$ and $q$ some integers such that $1 \leq n \leq 2^{m}$ and $1 \leq p \leq 2^{q}$, it is clear that $b(n, m)$ is topologically equivalent to $b(p, q)$ if and only if $q=m$. Hence, with the notation of Theorem 2.8 , we see that given $s, t \in(0,1)$, the sets $\Omega(t)$ and $\Omega(s)$ can be topologically equivalent only if they contain, for each fixed $m$, the same number of sets from the family $\{b(n, m)\}_{n=1}^{2^{m}}$. However if $s<t$ then there exist two positive integers $m$ and $n$ such that $s<\frac{2 n-1}{2^{m+1}}<t$, thus $b(n, m) \subset \Omega(s) \backslash \Omega(t)$ and therefore $\Omega(t)$ is not topologically equivalent to $\Omega(s)$.

\section{Applications to inverse magnetization problems}

\subsection{Solutions to Extremal Problem 1}

For $\boldsymbol{\mu}, \boldsymbol{\nu} \in \mathcal{M}\left(\mathbb{R}^{3}\right)$ with $\mathbf{f}_{\boldsymbol{\mu}}$ to denote the Radon-Nikodym derivative of $\boldsymbol{\mu}$ with respect to $|\boldsymbol{\nu}|$, we define for $|\boldsymbol{\nu}|$-a.e. $x$ :

$$
\mathbf{w}_{\boldsymbol{\mu}}^{\nu}(x):= \begin{cases}\frac{\mathbf{f}_{\mu}(x)}{\left|\mathbf{f}_{\mu}(x)\right|}, & \mathbf{f}_{\boldsymbol{\mu}}(x) \neq 0, \\ \mathbf{u}_{\boldsymbol{\nu}}(x), & \mathbf{f}_{\boldsymbol{\mu}}(x)=0 .\end{cases}
$$

We put $E=\mathbf{f}_{\boldsymbol{\mu}}^{-1}(0)$ and observe that

$$
\int \mathbf{w}_{\boldsymbol{\mu}}^{\nu} \cdot \mathrm{d} \boldsymbol{\nu}=\int_{E^{c}} \mathbf{w}_{\boldsymbol{\mu}}^{\nu} \cdot \mathbf{u}_{\boldsymbol{\nu}} \mathrm{d}|\boldsymbol{\nu}|+|\boldsymbol{\nu}|(E) .
$$

The next lemma provides a variational characterization of solutions to Extremal Problem 1.

Lemma 3.1. Let $S \subset \mathbb{R}^{3}$ be closed and suppose $\boldsymbol{\mu}, \boldsymbol{\nu} \in \mathcal{M}(S)^{3}$, with $\mathbf{w}_{\boldsymbol{\mu}}^{\nu}$ and $E$ as above. Then

$$
\|\boldsymbol{\mu}\|_{T V} \leq\|\boldsymbol{\mu}+t \boldsymbol{\nu}\|_{T V}, \text { for every } t>0,
$$

if and only if

$$
\int \mathrm{w}_{\mu}^{\nu} \cdot \mathrm{d} \boldsymbol{\nu} \geq 0
$$

Hence, $\|\boldsymbol{\mu}\|_{T V}=M_{S}(\boldsymbol{\mu})$ if and only if (3.4) holds for every $S$-silent $\boldsymbol{\nu} \in \mathcal{M}(S)^{3}$. The inequality (3.3) is strict for every $t>0$ if the inequality (3.4) is strict. 
Proof. Let $\boldsymbol{\mu}_{s}$ denote the singular part of $\boldsymbol{\mu}$ with respect to $|\boldsymbol{\nu}|$. Then, for $\epsilon>0$,

$$
\begin{aligned}
\|\boldsymbol{\mu}+\epsilon \boldsymbol{\nu}\|_{T V} & =\int\left|\mathbf{f}_{\boldsymbol{\mu}}+\epsilon \mathbf{u}_{\boldsymbol{\nu}}\right| \mathrm{d}|\boldsymbol{\nu}|+\left\|\boldsymbol{\mu}_{s}\right\|_{T V} \\
& =\int_{E^{c}}\left|\mathbf{f}_{\boldsymbol{\mu}}+\epsilon \mathbf{u}_{\nu}\right| \mathrm{d}|\boldsymbol{\nu}|+\epsilon|\boldsymbol{\nu}|(E)+\left\|\boldsymbol{\mu}_{s}\right\|_{T V} \\
& =\|\boldsymbol{\mu}\|_{T V}+\epsilon\left(\int_{E^{c}} \mathbf{w}_{\boldsymbol{\mu}}^{\nu} \cdot \mathbf{u}_{\boldsymbol{\nu}} \mathrm{d}|\boldsymbol{\nu}|+|\boldsymbol{\nu}|(E)\right)+o(\epsilon) \\
& =\|\boldsymbol{\mu}\|_{T V}+\epsilon \int \mathbf{w}_{\boldsymbol{\mu}}^{\nu} \cdot \mathrm{d} \boldsymbol{\nu}+o(\epsilon),
\end{aligned}
$$

where the above used that for $\mathbf{a}, \mathbf{b} \in \mathbb{R}^{3}, \mathbf{a} \neq 0$ and $|\mathbf{b}|=1$ (with $\mathbf{a}=\mathbf{f}_{\boldsymbol{\mu}}$ and $\mathbf{b}=\mathbf{u}_{\boldsymbol{\nu}}$ ),

$$
|\mathbf{a}+\epsilon \mathbf{b}|=|\mathbf{a}|\left(1+2 \epsilon \frac{\mathbf{a} \cdot \mathbf{b}}{|\mathbf{a}|^{2}}+\epsilon^{2} \frac{|\mathbf{b}|^{2}}{|\mathbf{a}|^{2}}\right)^{1 / 2}=|\mathbf{a}|+\epsilon \frac{\mathbf{a}}{|\mathbf{a}|} \cdot \mathbf{b}+\frac{1}{|\mathbf{a}|} \mathcal{O}\left(\epsilon^{2}\right),
$$

together with $|\boldsymbol{\nu}|\left(\left\{\mathbf{x}: 0<\left|\mathbf{f}_{\boldsymbol{\mu}}(\mathbf{x})\right|<\epsilon\right\}\right)=o(1)$ as $\epsilon \rightarrow 0$. Using the convexity of the TV-norm we have for $0<\epsilon \leq 1$ and $t>0$ :

$$
\|\boldsymbol{\mu}+t \epsilon \boldsymbol{\nu}\|_{T V}=\|(1-\epsilon) \boldsymbol{\mu}+\epsilon(\boldsymbol{\mu}+t \boldsymbol{\nu})\|_{T V} \leq(1-\epsilon)\|\boldsymbol{\mu}\|_{T V}+\epsilon\|\boldsymbol{\mu}+t \boldsymbol{\nu}\|_{T V}
$$

which implies

$$
t \frac{\|\boldsymbol{\mu}+\epsilon t \boldsymbol{\nu}\|_{T V}-\|\boldsymbol{\mu}\|_{T V}}{t \epsilon} \leq\|\boldsymbol{\mu}+t \boldsymbol{\nu}\|_{T V}-\|\boldsymbol{\mu}\|_{T V}
$$

If (3.4) holds, then it follows in view of (3.5) (with $t \epsilon$ instead of $\epsilon$ ) that the limit of the left-hand side of (3.6) is nonnegative when $\epsilon \rightarrow 0^{+}$, which implies (3.3). Conversely, if (3.3) holds then the left hand side of (3.6) is nonnegative and using (3.5) we can take the limit as $\epsilon \rightarrow 0^{+}$to obtain (3.4). That the inequality (3.3) is strict for every $t>0$ when the inequality (3.4) is strict follows immediately from the above computations.

We say that $\boldsymbol{\mu} \in \mathcal{M}(S)^{3}$ is carried by a set if that set has full $|\boldsymbol{\mu}|$-measure; i.e., the complement has $|\boldsymbol{\mu}|$ measure zero. Recall that a set $B \subset \mathbb{R}^{n}$ is purely 1-unrectifiable if $\mathcal{H}^{1}(E \cap B)=0$ for every 1-rectifiable set $E$. Clearly a set of $\mathcal{H}^{1}$-measure zero is purely 1-unrectifiable.

Theorem 3.2. Let $S \subset \mathbb{R}^{3}$ be slender and closed and suppose $\widetilde{\boldsymbol{\mu}} \in \mathcal{M}(S)^{3}$ is carried by a purely 1-unrectifiable set. Then $\widetilde{\boldsymbol{\mu}}$ is strictly $T V$-minimal. Moreover, if $\boldsymbol{\mu} \in \mathcal{M}(S)^{3}$ is $T V$-minimal on $S$, then so is $\boldsymbol{\mu}+\widetilde{\boldsymbol{\mu}}$.

Proof. Since $S$ is slender, any $S$-silent magnetization $\boldsymbol{\nu}$ is divergence-free. From the decomposition (2.11), we then have that $\boldsymbol{\nu}$ and $\widetilde{\boldsymbol{\mu}}$ are mutually singular since the latter is carried by a purely 1-unrectifiable set, showing that $\widetilde{\boldsymbol{\mu}}$ is strictly $T V$-minimal.

Next suppose $\boldsymbol{\mu} \in \mathcal{M}(S)^{3}$ satisfies $\|\boldsymbol{\mu}\|_{T V}=M_{S}(\boldsymbol{\mu})$ and $\boldsymbol{\nu} \in \mathcal{M}(S)^{3}$ be $S$-silent. Since $\boldsymbol{\nu}$ and $\widetilde{\boldsymbol{\mu}}$ are mutually singular, $d \widetilde{\boldsymbol{\mu}} / \mathrm{d}|\boldsymbol{\nu}|=0$ and thus, recalling definition (3.1), we see that $\mathbf{w}_{\boldsymbol{\mu}}^{\boldsymbol{\nu}}=\mathbf{w}_{\boldsymbol{\mu}+\tilde{\boldsymbol{\mu}}}^{\boldsymbol{\nu}},|\boldsymbol{\nu}|$-a.e. Lemma 3.1 then implies $\|\boldsymbol{\mu}+\widetilde{\boldsymbol{\mu}}\|_{T V}=M_{S}(\boldsymbol{\mu}+\widetilde{\boldsymbol{\mu}})$.

The first assertion of Theorem 3.2 sharpens Theorem 2.6 of [7] stating that a magnetization supported on a purely 1-unrectifiable set is strictly $T V$-minimal. In the case that $S$ is planar, this result can be strengthened by the following theorem. 
Theorem 3.3. Let $S \subset \mathbb{R}^{2} \times\{0\}$ be closed and suppose $\boldsymbol{\mu}$ is a magnetization carried by a Borel set $Z \subset S$ that satisfies

$$
\mathcal{H}^{1}(\Gamma \cap Z) \leq \mathcal{H}^{1}(\Gamma \backslash Z)
$$

for any rectifiable Jordan curve $\Gamma \subset S$. Then $\boldsymbol{\mu}$ is $T V$-minimal on $S$.

Let $\boldsymbol{\nu} \in \mathcal{M}(S)^{3}$ be $S$-silent whence $\boldsymbol{\nu}=\left(\boldsymbol{\nu}_{T}, 0\right)$ where $\boldsymbol{\nu}_{T}$ is divergence free, by Lemmas 2.1 and 2.2, and denote by $\Gamma_{n}^{t}$ the loop decomposition of $\boldsymbol{\nu}_{T}$ from Theorem 2.8. If $\|\boldsymbol{\mu}+\boldsymbol{\nu}\|_{T V}=\|\boldsymbol{\mu}\|_{T V}$, then for almost every $t$ equality holds in (3.7) whenever we substitute $\Gamma$ with $\Gamma_{n}^{t}$ and every $n \in \mathbb{N}$. In particular, $\boldsymbol{\mu}$ is strictly $T V$ minimal on $S$ if the inequality (3.7) is strict for every nondegenerate $\Gamma \subset S$, and then $\boldsymbol{\mu}+\tilde{\boldsymbol{\mu}}$ is also strictly $T V$-minimal when $\tilde{\boldsymbol{\mu}}$ is carried by a purely 1-unrectifiable set.

Proof. Let $\boldsymbol{\nu}$ be an $S$-silent magnetization with $\mathbf{f}_{\boldsymbol{\mu}}, \mathbf{w}_{\boldsymbol{\mu}}^{\boldsymbol{\nu}}$ as in (3.1), and recall $E=\mathbf{f}_{\boldsymbol{\mu}}^{-1}(0)$. Also let $\boldsymbol{\mu}_{s}$ denote the singular part of $\boldsymbol{\mu}$ with respect to $|\boldsymbol{\nu}|$. For $t \in \mathbb{R}$ and $n \in \mathbb{N}$, let $\boldsymbol{\tau}_{n}^{t}$ be as in Theorem 2.8 where $\boldsymbol{\nu}$ gets replaced by $\boldsymbol{\nu}_{T}$.

By assertion $(v)$ of Theorem 2.8, we know for a.e. $t \in \mathbb{R}$ and for every $n \in \mathbb{N}$ that $\mathbf{u}_{\nu}(x)=\left(\boldsymbol{\tau}_{n}^{t}(x), 0\right)$ for $\mathcal{H}^{1}$-a.e. $x \in \Gamma_{n}^{t}$. Note also that by $(i v)$ of Theorem $2.8, \mathbf{w}_{\boldsymbol{\mu}}^{\nu} \cdot\left(\boldsymbol{\tau}_{n}^{t}, 0\right)$ is $\mathcal{H}^{1}$-integrable on $\Gamma_{n}^{t}$ for every $n \in \mathbb{N}$ and a.e. $t \in \mathbb{R}$. Now, for every such $t$,

$$
\begin{aligned}
\int_{\Gamma_{n}^{t}} \mathbf{w}_{\boldsymbol{\mu}}^{\nu} \cdot\left(\boldsymbol{\tau}_{n}^{t}, 0\right) \mathrm{d} \mathcal{H}^{1} & =\int_{\Gamma_{n}^{t} \cap E^{c}} \mathbf{w}_{\boldsymbol{\mu}}^{\nu} \cdot\left(\boldsymbol{\tau}_{n}^{t}, 0\right) \mathrm{d} \mathcal{H}^{1}+\int_{\Gamma_{n}^{t} \cap E} \mathbf{u}_{\boldsymbol{\nu}} \cdot\left(\boldsymbol{\tau}_{n}^{t}, 0\right) \mathrm{d} \mathcal{H}^{1} \\
& =\int_{\Gamma_{n}^{t} \cap E^{c}} \mathbf{w}_{\boldsymbol{\mu}}^{\nu} \cdot\left(\boldsymbol{\tau}_{n}^{t}, 0\right) \mathrm{d} \mathcal{H}^{1}+\mathcal{H}^{1}\left(\Gamma_{n}^{t} \cap E\right) \\
& \geq-\mathcal{H}^{1}\left(\Gamma_{n}^{t} \cap E^{c}\right)+\mathcal{H}^{1}\left(\Gamma_{n}^{t} \cap E\right) .
\end{aligned}
$$

From (2.14) we have

$$
0=\int_{Z^{c}}\left|\mathbf{f}_{\boldsymbol{\mu}}\right| \mathrm{d}|\boldsymbol{\nu}|=\int_{\mathbb{R}} \sum_{n \in \mathbb{N}}\left(\int_{Z^{c}}\left|\mathbf{f}_{\boldsymbol{\mu}}\right| \mathrm{d}\left(\mathcal{H}^{1}\left\lfloor\Gamma_{n}^{t}\right)\right) \mathrm{d} t .\right.
$$

Observing that $\left|\mathbf{f}_{\boldsymbol{\mu}}(x)\right|>0$ for $x \in E^{c}$, the above equation implies that the $\mathcal{L}_{1}$-measure of

$$
T_{0}:=\left\{t \in \mathbb{R} \mid \exists n \in \mathbb{N}: \mathcal{H}^{1}\left(\Gamma_{n}^{t} \cap E^{c} \cap Z^{c}\right) \neq 0\right\}
$$

is zero; that is, $\mathcal{H}^{1}\left(\Gamma_{n}^{t} \cap E^{c} \cap Z^{c}\right)=0$ for a.e. $t$. Thus, by (3.8) we get that

$$
\int_{\Gamma_{n}^{t}} \mathbf{w}_{\boldsymbol{\mu}}^{\nu} \cdot\left(\boldsymbol{\tau}_{n}^{t}, 0\right) \mathrm{d} \mathcal{H}^{1} \geq-\mathcal{H}^{1}\left(\Gamma_{n}^{t} \cap Z\right)+\mathcal{H}^{1}\left(\Gamma_{n}^{t} \backslash Z\right) \geq 0
$$

where the last inequality follows from the condition (3.7). Therefore, by (2.13),

$$
\int_{\mathbb{R}^{2}} \mathbf{w}_{\boldsymbol{\mu}}^{\nu} \cdot \mathrm{d} \boldsymbol{\nu}=\int_{\mathbb{R}} \sum_{n \in N^{t}}\left(\int_{\mathbb{R}^{2}} \mathbf{w}_{\boldsymbol{\mu}}^{\nu} \cdot\left(\boldsymbol{\tau}_{n}^{t}, 0\right) \mathrm{d}\left(\mathcal{H}^{1}\left\lfloor\Gamma_{n}^{t}\right)\right) \mathrm{d} t \geq 0\right.
$$

and, hence, Lemma 3.1 gives us $\|\boldsymbol{\mu}\|_{T V} \leq\|\boldsymbol{\mu}+\boldsymbol{\nu}\|_{T V}$. Moreover, if there is a set of positive measure $E \subset \mathbb{R}$ such that for every $t \in E$ there exists an $n$ for which the rightmost inequality in (3.9) is strict, then the inequality in (3.10) is also strict. Finally, (3.7) is invariant upon adding a purely 1-unrectifiable set to $Z$. 
Corollary 3.4. Let $S \subset \mathbb{R}^{2} \times\{0\}$ be closed and suppose $\boldsymbol{\mu}$ is a magnetization carried by a Borel set $Z \subset S$ that is contained in a purely 1-unrectifiable set plus a countable union $\bigcup_{k \in K} L_{k}$ where the $L_{k}$ are disjoint line segments such that the distance from any $L_{k}$ to any $L_{j}, j \neq k$, is greater than or equal to the length of $L_{k}$. Then (3.7) holds for any rectifiable Jordan curve $\Gamma$, and thus $\boldsymbol{\mu}$ is TV-minimal on $S$. Moreover, if the distance from any $L_{k}$ to any $L_{j}, j \neq k$, is strictly greater than the length of $L_{k}$, then (3.7) is strict and $\boldsymbol{\mu}$ is strictly $T V$-minimal on $S$.

Proof. By the last assertion of Theorem 3.3, it is enough to assume $Z$ is contained in a countable union of line segments with the aforementioned properties. Let $\Gamma$ be a rectifiable Jordan curve oriented by a parametrization $\gamma$. Without loss of generality we may assume that $Z \cap L_{k} \neq \emptyset$ for all $k \in K$. If $K=\{1\}$ is a singleton, then (since $L_{1}$ is a line segment)

$$
\mathcal{H}^{1}(\Gamma \cap Z) \leq \mathcal{H}^{1}\left(\Gamma \cap L_{1}\right)<\mathcal{H}^{1}\left(\Gamma \backslash L_{1}\right) \leq \mathcal{H}^{1}(\Gamma \backslash Z) .
$$

Otherwise, for each $k \in K$ there is some directed sub-arc $\Gamma_{k} \subset \Gamma$ with initial point in $L_{k}$, end point in some $L_{j}$ for $j \neq k$, and interior in the complement of $\bigcup_{\ell \neq k} L_{\ell}$. Note that for $j \neq k \in K$, the interiors of $\Gamma_{k}$ and $\Gamma_{j}$ are disjoint, and that $\mathcal{H}^{1}\left(\Gamma \cap L_{k}\right) \leq \mathcal{H}^{1}\left(\Gamma_{k}\right)$ by assumption. Also note that this inequality is strict under the final assumption. Thus,

$$
\mathcal{H}^{1}(\Gamma \cap Z) \leq \sum_{k \in K} \mathcal{H}^{1}\left(\Gamma \cap L_{k}\right) \leq \sum_{k \in K} \mathcal{H}^{1}\left(\Gamma_{k}\right) \leq \mathcal{H}^{1}(\Gamma \backslash Z)
$$

where the second inequality is strict under the last assumption.

We next characterize the space of $S$-silent magnetizations when $S$ contains only a finite number of Jordan curves. First we consider the class of closed $S \subset \mathbb{R}^{2}$ that contain no rectifiable Jordan curve at all, and hence, cannot hold nontrivial silent magnetizations. We call such $S$ tree-like. Note that any closed purely 1-unrectifiable set is tree-like, but the converse is not true. We also note that a tree-like set may contain a Jordan curve, such as the Koch curve, which is not rectifiable. As a consequence of Theorem 2.8 we obtain the following result.

Lemma 3.5. Let $S$ be a closed subset of $\mathbb{R}^{2} \times\{0\}$. If $\boldsymbol{\mu} \in \mathcal{M}(S)^{3}$ is nonzero and $S$-silent, then the support of $\boldsymbol{\mu}$ contains a rectifiable Jordan curve. Hence, if $S$ is tree-like the only $S$-silent magnetization is the zero magnetization.

Proof. Since $S \subset \mathbb{R}^{2} \times\{0\}$, it is slender and hence $S$-silent magnetizations are divergence free. The lemma now follows from Theorem 2.8 .

For a closed set $S \subset \mathbb{R}^{2} \times\{0\}$, let $\Sigma(S)$ denote the linear subspace of $\mathcal{M}(S)^{3}$ consisting of $S$-silent sources. The previous lemma shows that $\Sigma(S)$ is the trivial subspace when $S$ is tree-like. Next, we present a theorem which provides sufficient conditions for $\Sigma(S)$ to be finite dimensional and generalizes the second assertion of Lemma 3.5 when $\mathcal{H}^{1}(S)$ is finite. First, we record an elementary lemma used in the proof Theorem 3.7.

Lemma 3.6. If $\left\{\Gamma_{i}\right\}_{i=1}^{N}$ is a family of Jordan curves in $\mathbb{R}^{2}$ such that for any $i$, the union $\bigcup_{j \neq i} \Gamma_{j}$ does not contain $\Gamma_{i}$, then $\mathbb{R}^{2} \backslash \bigcup_{i=1}^{N} \Gamma_{i}$ contains at least $N+1$ connected components.

Proof. The proof will be done by induction. The case $N=1$ is just the Jordan curve theorem. Assume that $\mathbb{R}^{2} \backslash \bigcup_{i=1}^{N-1} \Gamma_{i}$ contains at least $N$ connected (necessarily open) components. By connectness, any connected (also necessarily open) component of $\mathbb{R}^{2} \backslash \bigcup_{i=1}^{N} \Gamma_{i}$ is contained in a connected component of $\mathbb{R}^{2} \backslash \bigcup_{i=1}^{N-1} \Gamma_{i}$, conversely any connected component of $\mathbb{R}^{2} \backslash \bigcup_{i=1}^{N-1} \Gamma_{i}$ must contain at least one connected component of $\mathbb{R}^{2} \backslash \bigcup_{i=1}^{N} \Gamma_{i}$. Since $\Gamma_{N} \not \subset \bigcup_{i=1}^{N-1} \Gamma_{i}$, there exists a connected component $U$ of $\mathbb{R}^{2} \backslash \bigcup_{i=1}^{N-1} \Gamma_{i}$ such that $U \cap \Gamma_{N} \neq \emptyset$. Then, by the Jordan curve theorem, $U \cap \mathbb{R}^{2} \backslash \bigcup_{i=1}^{N} \Gamma_{i}$ is disconnected. Hence, at least two connected components 
of $\mathbb{R}^{2} \backslash \bigcup_{i=1}^{N} \Gamma_{i}$ are contained in $U$; therefore, it follows that $\mathbb{R}^{2} \backslash \bigcup_{i=1}^{N} \Gamma_{i}$ has a larger number of connected components than $\mathbb{R}^{2} \backslash \bigcup_{i=1}^{N-1} \Gamma_{i}$ which, in turn, contains at least $N$. This completes the proof.

Theorem 3.7. Let $S \subset \mathbb{R}^{2} \times\{0\}$ be closed with empty interior and such that the number $n$ of bounded connected components of $\mathbb{R}^{2} \times\{0\} \backslash S$ is finite. Then the dimension of $\Sigma(S)$ is less than or equal to $n$ with equality if $\mathcal{H}^{1}(S)$ is finite.

Proof. Let $S^{\prime} \subset S$ be any union of finitely many rectifiable Jordan curves contained in $S$ and let $m$ be the number of bounded connected (necessarily open) components of $\mathbb{R}^{2} \backslash S^{\prime}$. For any $A \subset \mathbb{R}^{2}$, let $\mathfrak{C}(A)$ denote the family of connected components of $A$ and let $\mathfrak{B}(A) \subset \mathfrak{C}(A)$ be the bounded ones. Since $\left(\mathbb{R}^{2} \backslash S\right) \cup\left(S \backslash S^{\prime}\right)=\left(\mathbb{R}^{2} \backslash S^{\prime}\right)$, for every $C \in \mathfrak{C}\left(\mathbb{R}^{2} \backslash S\right)$, there exists a unique $f(C) \in \mathfrak{C}\left(\mathbb{R}^{2} \backslash S^{\prime}\right)$ such that $C \subset f(C)$. Now, since the set $S \backslash S^{\prime}$ has empty interior, for every $D \in \mathfrak{B}\left(\mathbb{R}^{2} \backslash S^{\prime}\right)$, we have that $D \not \subset\left(S \backslash S^{\prime}\right)$, hence $D \cap C \neq \emptyset$ for some $C \in \mathfrak{C}\left(\mathbb{R}^{2} \backslash S\right)$ and thus, for any such $C, C \subset D$ whence $C$ is bounded, and $f(C)=D$. Let $f_{\mathfrak{B}}$ be the restriction of $f$ to $\mathfrak{B}\left(\mathbb{R}^{2} \backslash S\right)$. As a consequence of the above, the image of $f_{\mathfrak{B}}$ contains $\mathfrak{B}\left(\mathbb{R}^{2} \backslash S^{\prime}\right)$ and therefore $n \geq m$. Lemma 3.6 now implies that there exists a family of at most $n$ rectifiable Jordan curves whose union contains all rectifiable Jordan curves contained in $S$. Therefore, we can assume that $S^{\prime}$ is the union all rectifiable Jordan curves contained in $S$. Note that $S^{\prime}$ is closed and $\mathcal{H}^{1}\left(S^{\prime}\right)$ is finite.

From Theorem 2.8 it follows that $\Sigma(S)=\Sigma\left(S^{\prime}\right)$. Thus, showing that $\operatorname{dim} \Sigma\left(S^{\prime}\right)=m$ will prove our theorem. Let $\left\{E_{i}\right\}_{i=1}^{m}$ be the family of bounded connected components of $\mathbb{R}^{2} \backslash S^{\prime}$. Note that each $E_{i}$ is of finite perimeter since $\mathcal{H}^{1}\left(S^{\prime}\right)$ is finite (see for example [15], Thm. 5.23 and then Thm. 5.15). Let $\boldsymbol{\ell}_{i}:=\mathfrak{R} \nabla \chi_{E_{i}}$ for $i=1, \ldots m$. By Lemmas 2.1 and 2.2, each $\boldsymbol{\ell}_{i}$ is $S^{\prime}$-silent. To show that $\left\{\boldsymbol{\ell}_{i}\right\}_{i=1}^{m}$ generates $\Sigma\left(S^{\prime}\right)$, it is sufficient by Theorem 2.8 to prove that for any rectifiable Jordan curve $\Gamma \subset S^{\prime}$ with parametrization $\boldsymbol{\gamma}$, the magnetization $\mathbf{R}_{\gamma}$ defined by (2.1) is in the span of the $\boldsymbol{\ell}_{i}$ 's.

Using the Jordan curve theorem we can see that for any $E_{i}$ such that $\operatorname{int}(\Gamma) \cap E_{i} \neq \emptyset$ we have that $E_{i} \subset$ $\operatorname{int}(\Gamma)$. Hence there exists a $J \subset\{1, \ldots, m\}$ such that $\bigcup_{i \in J} E_{i} \subset \operatorname{int}(\Gamma) \subset S^{\prime} \cup \bigcup_{i \in J} E_{i}$ and since $\mathcal{L}_{2}\left(S^{\prime}\right)=0$ while the $E_{i}$ 's are disjoint, then

$$
\begin{aligned}
\pm \mathbf{R}_{\gamma} & =\mathfrak{R} \nabla \chi_{\operatorname{int}(\Gamma)}=\mathfrak{R} \nabla \chi_{\bigcup_{i \in J} E_{i}} \\
& =\sum_{i \in J} \mathfrak{R} \nabla \chi_{E_{i}}=\sum_{i \in J} \ell_{i},
\end{aligned}
$$

where the first equality comes from the remark after (2.7), equation (2.6) and Lemma 2.7.

To show linearly independence, assume that $\sum_{i=1}^{m} c_{i} \ell_{i}=0$ where $c_{i} \in \mathbb{R}, i=1, . ., m$. Since $0=$ $\sum_{i=1}^{m} c_{i} \mathfrak{R} \nabla \chi_{E_{i}}=\mathfrak{R} \nabla\left(\sum_{i=1}^{m} c_{i} \chi_{E_{i}}\right)$, thus $\sum_{i=1}^{m} c_{i} \chi_{E_{i}}$ is a constant but since the $E_{i}$ 's are bounded and disjoint then each $c_{i}=0$ and hence the $\boldsymbol{\ell}_{i}$ 's are indeed linearly independent.

\subsection{Regularization by penalizing the total variation}

Let $S \subset \mathbb{R}^{2} \times\{0\}$ and $Q \subset \mathbb{R}^{3}$ be closed and positively separated. For $\boldsymbol{\mu} \in \mathcal{M}(S)^{3}$ and $v$ a unit vector in $\mathbb{R}^{3}$, the component of the magnetic field $\mathbf{b}(\boldsymbol{\mu})$ in the direction $v$ at $x \notin S$ is given, in view of (1.1), by

$$
b_{v}(\boldsymbol{\mu})(x):=v \cdot \mathbf{b}(\boldsymbol{\mu})(x)=-\frac{\mu_{0}}{4 \pi} \int \mathbf{K}_{v}(x-y) \cdot \mathrm{d} \boldsymbol{\mu}(y),
$$

where

$$
\mathbf{K}_{v}(x)=\frac{v}{|x|^{3}}-3 x \frac{v \cdot x}{|x|^{5}}=\nabla\left(\frac{v \cdot x}{|x|^{3}}\right) .
$$


Consider a finite, positive Borel measure $\rho$ with support contained in $Q$ and let $A: \mathcal{M}(S)^{3} \rightarrow L^{2}(Q, \rho)$ be the so-called forward operator defined by

$$
A(\boldsymbol{\mu})(x):=b_{v}(\boldsymbol{\mu})(x), \quad x \in Q .
$$

The adjoint operator $A^{*}$ is then given by (see [7], Sect. 3)

$$
A^{*}(\Psi)(x):=-\mu_{0} \nabla\left(\nabla U^{\rho, \psi} \cdot v\right)(x), \quad U^{\rho, \psi}(x)=-\frac{1}{4 \pi} \int \frac{\Psi(y)}{|x-y|} \mathrm{d} \rho(y) .
$$

Since $Q$ and $S$ are positively separated it follows from the harmonicity of $K_{v}$ that $A^{*}(\Psi) \in C_{0}(S)^{3}$ and thus $A^{*}:\left(L^{2}(Q, \rho)\right)^{*} \sim L^{2}(Q, \rho) \rightarrow C_{0}(S)^{3} \subset\left(\mathcal{M}(S)^{3}\right)^{*}$. Note the kernel of the forward operator $A$ contains all $S$-silent magnetizations. In the case this kernel consists exactly of $S$-silent magnetizations, we say that $A$ is $S$-sufficient. It follows from Lemmma 2.3 of [7] and the discussion thereafter that $A$ is $S$-sufficient when $S \subset \mathbb{R}^{2} \times\{0\}$ and $Q \subset \mathbb{R}^{3}$ are positively separated closed sets and for some complete real analytic surface $\mathcal{A} \subset \mathbb{R}^{3} \backslash S$ we have:

1. $S$ and $\mathcal{A}$ are positively separated;

2. $S$ lies entirely within one connected component of $\mathbb{R}^{3} \backslash \mathcal{A}$;

3. $Q \cap \mathcal{A}$ has Hausdorff dimension strictly greater than 1 in each connected component of $\mathbb{R}^{3} \backslash S$;

4. $\operatorname{supp} \rho=Q$.

For $\boldsymbol{\mu} \in \mathcal{M}(S)^{3}, f \in L^{2}(Q, \rho)$, and $\lambda>0$, recall from (1.4) the definition of $\mathcal{F}_{f, \lambda}$, and from (1.5) the notation $\boldsymbol{\mu}_{\boldsymbol{\lambda}} \in \mathcal{M}(S)^{3}$ to designate a minimizer of $\mathcal{F}_{f, \lambda}$. As a second application of our results in Section 2.3, we prove:

Theorem 3.8. Let $S$ be a closed subset of $\mathbb{R}^{2} \times\{0\}, Q \subset \mathbb{R}^{3}$ be a closed set and $\rho \in \mathcal{M}(Q)$ be such that the forward operator $A$ defined in (3.13) is $S$-sufficient. For $f \in L^{2}(Q, \rho)$ and $\lambda>0$, the solution to (1.5) is unique.

Proof. It is well known (see e.g. [10], Prop. 3.6) that $\boldsymbol{\mu}_{\lambda} \in \mathcal{M}(S)^{3}$ is a minimizer of $\mathcal{F}_{f, \lambda}$ if and only if:

$$
\begin{array}{ll}
A^{*}\left(f-A \boldsymbol{\mu}_{\lambda}\right) & =\frac{\lambda}{2} \mathbf{u}_{\boldsymbol{\mu}_{\lambda}} \quad\left|\boldsymbol{\mu}_{\lambda}\right| \text {-a.e. and } \\
\left|A^{*}\left(f-A \boldsymbol{\mu}_{\lambda}\right)\right| & \leq \frac{\lambda}{2} \quad \text { everywhere on } S .
\end{array}
$$

Moreover, it follows from the strict convexity of the $L^{2}$-norm that $\boldsymbol{\mu}_{\lambda}^{\prime} \in \mathcal{M}(S)^{3}$ is another solution if and only if $A\left(\boldsymbol{\mu}_{\lambda}^{\prime}-\boldsymbol{\mu}_{\lambda}\right)=0$.

Assume for a contradiction that $\boldsymbol{\mu}_{\lambda}$ and $\boldsymbol{\mu}_{\lambda}^{\prime}$ are two distinct minimizers in (1.5) and let $\boldsymbol{\mu}:=\boldsymbol{\mu}_{\lambda}^{\prime}-\boldsymbol{\mu}_{\lambda}$. As $\boldsymbol{\mu}_{\lambda}^{\prime}-\boldsymbol{\mu}_{\lambda}=\boldsymbol{\mu}$ is absolutely continuous with respect to $|\boldsymbol{\mu}|$, the Lebesgue decompositions of $\boldsymbol{\mu}_{\lambda}$ and $\boldsymbol{\mu}_{\lambda}^{\prime}$ with respect to $|\boldsymbol{\mu}|$ must have the same singular term. That is, these decompositions are necessarily of the form

$$
\mathrm{d} \boldsymbol{\mu}_{\lambda}=\gamma \mathrm{d}|\boldsymbol{\mu}|+\mathrm{d} \boldsymbol{\nu}, \quad \mathrm{d} \boldsymbol{\mu}_{\lambda}^{\prime}=\gamma^{\prime} \mathrm{d}|\boldsymbol{\mu}|+\mathrm{d} \boldsymbol{\nu}
$$

where $|\boldsymbol{\nu}|$ is singular with respect to $|\boldsymbol{\mu}|$ and $\boldsymbol{\gamma}, \boldsymbol{\gamma}^{\prime}$ are $|\boldsymbol{\mu}|$-integrable $\mathbb{R}^{3}$-valued functions.

Put for simplicity $\psi=(2 / \lambda)\left(f-A\left(\boldsymbol{\mu}_{\lambda}\right)\right)=(2 / \lambda)\left(f-A\left(\boldsymbol{\mu}_{\lambda}^{\prime}\right)\right)$. Thanks to (3.15) we know that $\mathbf{u}_{\boldsymbol{\mu}_{\lambda}}=A^{*} \psi$ and $\mathbf{u}_{\boldsymbol{\mu}_{\lambda}^{\prime}}=A^{*} \psi, \boldsymbol{\mu}_{\lambda}$ and $\boldsymbol{\mu}_{\lambda}^{\prime}$-a.e. respectively. Now, since d $\left|\boldsymbol{\mu}_{\lambda}\right|=|\gamma| \mathrm{d}|\boldsymbol{\mu}|+\mathrm{d}|\boldsymbol{\nu}|$ and $\mathrm{d}\left|\boldsymbol{\mu}_{\lambda}^{\prime}\right|=\left|\gamma^{\prime}\right| \mathrm{d}|\boldsymbol{\mu}|+\mathrm{d}|\boldsymbol{\nu}|$, we have that

$$
\mathbf{u}_{\boldsymbol{\mu}} \mathrm{d}|\boldsymbol{\mu}|=\mathrm{d} \boldsymbol{\mu}=\mathbf{u}_{\boldsymbol{\mu}_{\lambda}^{\prime}} \mathrm{d}\left|\boldsymbol{\mu}_{\lambda}^{\prime}\right|-\mathbf{u}_{\boldsymbol{\mu}_{\lambda}} \mathrm{d}\left|\boldsymbol{\mu}_{\lambda}\right|=A^{*} \psi \mathrm{d}\left|\boldsymbol{\mu}_{\lambda}^{\prime}\right|-A^{*} \psi \mathrm{d}\left|\boldsymbol{\mu}_{\lambda}\right|=A^{*} \psi\left(\left|\boldsymbol{\gamma}^{\prime}\right|-|\gamma|\right) \mathrm{d}|\boldsymbol{\mu}|
$$

Therefore $\mathbf{u}_{\boldsymbol{\mu}}=A^{*} \psi\left(\left|\boldsymbol{\gamma}^{\prime}\right|-|\boldsymbol{\gamma}|\right)$ at $|\boldsymbol{\mu}|$-a.e point, and since $\left|A^{*} \psi\right|=1$ on the supports of $\boldsymbol{\mu}_{\lambda}$ and $\boldsymbol{\mu}_{\lambda}^{\prime}$ it holds that $\mathbf{u}_{\mu}(x)= \pm_{x} A^{*} \psi(x)$ for $|\boldsymbol{\mu}|$-a.e. $x$, where the choice of sign $\pm_{x}$ has a subscript $x$ to indicate that it may vary with $x$. 
From the $S$-sufficiency of $A$ we know that $\boldsymbol{\mu}$ is $S$-silent. Also, by Corollary 4.2 of [7] (take $\mathcal{B}=\mathbb{R}^{2} \times\{0\}$ there), the supports of $\boldsymbol{\mu}_{\lambda}$ and $\boldsymbol{\mu}_{\lambda}^{\prime}$ are contained in a finite collection of points and analytic arcs. In particular, there are only finitely many rectifiable Jordan curves contained in the support of $\boldsymbol{\mu}$ and they are all piecewise analytic. Thus, applying Theorem 2.8 to $\boldsymbol{\mu}$, we find there are finitely many piecewise analytic oriented Jordan curves $\Gamma_{1}, \cdots, \Gamma_{N}$ with respective unit tangent vector fields $\boldsymbol{\tau}_{1}, \cdots, \boldsymbol{\tau}_{n}$, and strictly positive real numbers $a_{1}, \cdots, a_{N}$ such that $\boldsymbol{\tau}_{m}=\boldsymbol{\tau}_{n}$ on $\Gamma_{m} \cap \Gamma_{n}, \mathcal{H}^{1}$-a.e. and

$$
\mathrm{d} \boldsymbol{\mu}=\sum_{n=1}^{N} a_{n} \boldsymbol{\tau}_{n} \mathrm{~d}\left(\mathcal{H}^{1}\left\lfloor\Gamma_{n}\right) .\right.
$$

In particular, $\mathrm{d}|\boldsymbol{\mu}|=\sum_{n=1}^{N} a_{n} \mathrm{~d}\left(\mathcal{H}^{1}\left\lfloor\Gamma_{n}\right)\right.$ and $\boldsymbol{\tau}_{n}(x)=\mathbf{u}_{\boldsymbol{\mu}}(x)= \pm_{x} A^{*} \psi(x)$, for $|\boldsymbol{\mu}|$-a.e. $x$, hence $\mathcal{H}^{1}$-a.e., on $\Gamma_{n}$.

Fix $n$ and let $E$ be an analytic sub-arc of $\Gamma_{n}$. Being the unit tangent to an oriented analytic arc, $\boldsymbol{\tau}_{n}(x)$ must be an analytic function of $x \in E$, and so is $A^{*} \psi(x)$ by the real analyticity of $A^{*} \psi, c f$. (3.14). Hence, either $\boldsymbol{\tau}_{n}=A^{*} \psi$ or $\boldsymbol{\tau}_{n}=-A^{*} \psi$ everywhere on $E$. Therefore, $E$ is a subset of a trajectory of the autonomous differential equation $\dot{x}=A^{*} \psi(x)$. Moreover, since $E$ is bounded and percursed at unit speed, the corresponding trajectory extends beyond the endpoints of $E$, and since two distinct trajectories cannot intersect we conclude that $\Gamma_{n}$ is smooth and constitutes a single, periodic trajectory. This, however, is impossible because $A^{*} \psi$ is a gradient vector field, by (3.14).

When $S$ is planar and EP-1 has a unique solution, Theorem 4.3 from [7] and Theorem 3.8 together imply the following corollary.

Corollary 3.9. Let $S \subset \mathbb{R}^{2} \times\{0\}$ be closed, the forward operator $A$ be $S$-sufficient, and $\boldsymbol{\mu}_{0} \in \mathcal{M}(S)^{3}$. Set $f=A \boldsymbol{\mu}_{0}$ and, for $e \in L^{2}(Q, \rho)$, set $f_{e}:=f+e$. For $\lambda>0$, there is a unique minimizer $\boldsymbol{\mu}_{\lambda, e}$ of (1.4) where $f$ gets replaced by $f_{e}$.

If $\|\boldsymbol{\mu}\|_{T V}>\left\|\boldsymbol{\mu}_{0}\right\|_{T V}$ for any magnetization $\boldsymbol{\mu}$ that is $S$-equivalent to $\boldsymbol{\mu}_{0}$, then $\boldsymbol{\mu}_{\lambda, e}$ (resp. $\left.\left|\boldsymbol{\mu}_{\lambda, e}\right|\right)$ converges to $\boldsymbol{\mu}_{0}$ (resp. $\left.\left|\boldsymbol{\mu}_{0}\right|\right)$ in the narrow sense as $\lambda \rightarrow 0$ and $\|e\|_{L^{2}(Q)} / \sqrt{\lambda} \rightarrow 0$.

Theorems 3.2 and 3.3, Corollary 3.4, and Lemma 3.5 give sufficient conditions for the uniqueness of solutions to EP-1. Hence, if $\boldsymbol{\mu}_{0} \in \mathcal{M}(S)^{3}$ is carried by a set $Z \subset S \subset \mathbb{R}^{2} \times\{0\}$, then we may apply the above corollary under the following conditions:

(a) $\mathcal{H}^{1}(\Gamma \cap Z)<\mathcal{H}^{1}(\Gamma \backslash Z)$ for any rectifiable Jordan curve $\Gamma \subset S$, or

(b) $Z \subset W \cup \bigcup_{k \in K} L_{k}$ where $W \subset S$ is purely 1-unrectifiable and the $L_{k}$ are disjoint line segments such that the distance from any $L_{k}$ to any $L_{j}, j \neq k$, is greater than the length of $L_{k}$, or

(c) $S$ is tree-like.

In particular, it follows from condition (b) that Corollary 3.9 applies when $\boldsymbol{\mu}_{0}$ is carried by a countable collection of points and sufficiently separated line segments.

We conclude with an example.

Example 3.10. Let $v_{0}=v_{4}=(-1,-1), v_{1}=(1,-1), v_{2}=(1,1)$, and $v_{3}=(-1,1)$ denote the vertices of the square $[-1,1]^{2}$ and let $\gamma_{i}$ denote the arclength parametrization of the directed line segment from $v_{i}$ to $v_{i+1}$ for $i=0,1,2,3$. Let $\boldsymbol{\mu}_{1}=\mathbf{R}_{\gamma_{0}}+\mathbf{R}_{\boldsymbol{\gamma}_{2}}$ and $\boldsymbol{\mu}_{2}=-\mathbf{R}_{\boldsymbol{\gamma}_{1}}-\mathbf{R}_{\boldsymbol{\gamma}_{3}}$ and let $S$ be the square [-2,2] $]^{2}$. By Corollary 3.4 both $\boldsymbol{\mu}_{1}$ and $\boldsymbol{\mu}_{2}$ are $T V$-minimal on $S$. However, $\boldsymbol{\mu}_{1}$ and $\boldsymbol{\mu}_{2}$ are not strictly $T V$-minimal since $\boldsymbol{\mu}_{1}-\boldsymbol{\mu}_{2}$ is $S$-silent by Lemma 2.2 and Theorem 2.8, showing that $\boldsymbol{\mu}_{1}$ and $\boldsymbol{\mu}_{2}$ are $S$-equivalent. Clearly, any convex combination $(1-$ a) $\boldsymbol{\mu}_{1}+\alpha \boldsymbol{\mu}_{2}, \alpha \in[0,1]$, is also $S$-equivalent to $\boldsymbol{\mu}_{1}$ and $T V$-minimal on $S$. In fact, any $T V$-minimal magnetization which is $S$-equivalent to $\boldsymbol{\mu}_{1}$ is of this form. Indeed, taking $\boldsymbol{\mu}=\boldsymbol{\mu}_{1}$ and $Z=\operatorname{supp} \boldsymbol{\mu}_{1}$, in (3.7), the only $\Gamma$ that makes this inequality an equality is the boundary of $[-1,1]^{2}$. Hence, by Theorem 3.3, any $T V$-minimal magnetization is of the form $\boldsymbol{\mu}_{1}+s\left(\boldsymbol{\mu}_{2}-\boldsymbol{\mu}_{1}\right)$ for some $s \in \mathbb{R}$ and minimality of the total variation forces $0 \leq s \leq 1$. 
If we take $Q=[-1,1]^{2} \times\{1\}$ and $\rho=\mathcal{L}_{2}\lfloor Q$ then the forward operator $A$ is $S$-sufficient. Assume further that in (3.13), $v$ is the unit vector $(0,0,1)^{T}$. Recall the notation $\mathfrak{R}$ for the rotation by $\pi / 2$, and for any $\boldsymbol{\mu} \in \mathcal{M}(S)^{2}$ let $\tilde{\mathfrak{R}} \boldsymbol{\mu}$ be defined as $\langle\tilde{\mathfrak{R}} \boldsymbol{\mu}, \boldsymbol{\phi}\rangle:=\left\langle\boldsymbol{\mu}, \mathfrak{R}^{-1} \circ \boldsymbol{\phi} \circ \mathfrak{R}\right\rangle$, for any $\boldsymbol{\phi} \in C_{c}\left(S, \mathbb{R}^{2}\right)$. Observe that $\mathfrak{R}^{-1}=\mathfrak{R}^{t}$, which implies that for any parametrized rectifiable curve $\gamma$ we have $\tilde{\mathfrak{R}} \mathbf{R}_{\boldsymbol{\gamma}}=\mathbf{R}_{\mathfrak{R} \gamma}$ and hence, $\tilde{\mathfrak{R}} \boldsymbol{\mu}_{1}=-\boldsymbol{\mu}_{2}$ as well as $\tilde{\mathfrak{R}} \boldsymbol{\mu}_{2}=-\boldsymbol{\mu}_{1}$. Also note that $A \tilde{\mathfrak{R}} \boldsymbol{\mu}=(A \boldsymbol{\mu}) \circ \mathfrak{R}^{-1}$, since $\mathfrak{R}$ commutes with the first two components of $\mathbf{K}_{v}$ from (3.12). Now, with the notation of Corollary 3.9, set $\boldsymbol{\mu}_{0}=\left(\boldsymbol{\mu}_{1}+\boldsymbol{\mu}_{2}\right) / 2$, so that $f:=A \boldsymbol{\mu}_{\mathbf{0}}$ satisfies $f=-f \circ \mathfrak{R}^{-1}$. If in addition the noise $e$ satisfies $e=-e \circ \mathfrak{R}^{-1}$, then $f_{e}=-f_{e} \circ \mathfrak{R}^{-1}$ as well. In this case, we get that $\mathcal{F}_{f_{e}, \lambda} \circ(-\tilde{\mathfrak{R}})=\mathcal{F}_{f_{e}, \lambda}$ and thus Theorem 3.8 implies that $-\tilde{\mathfrak{R}} \boldsymbol{\mu}_{\lambda, e}=\boldsymbol{\mu}_{\lambda, e}$ for every $\lambda>0$. Now, we know that any weak-* limit of minimizers of EP-2 is $T V$-minimal, provided that both $\lambda$ and $\left\|e \lambda^{-1 / 2}\right\|_{L^{2}(Q, \rho)}$ tend to 0 (see [11], Thms. 2\&5). Because the limit should be invariant under $-\tilde{\mathfrak{R}}$ by what precedes, it must be equal to $\left(\boldsymbol{\mu}_{1}+\boldsymbol{\mu}_{2}\right) / 2$. In particular, we get global weak-* convergence of both $\boldsymbol{\mu}_{\lambda, e}$ and $\left|\boldsymbol{\mu}_{\lambda, e}\right|$ in this example, as long as $e=-e \circ \Re^{-1}$; i.e., when the noise $e$ has the same symmetry as the data.

\section{REFERENCES}

[1] L. Ambrosio, V. Caselles, S. Masnou and J.-M. Morel, Connected components of sets of finite perimeter and applications to image processing. J. Eur. Math. Soc. (JEMS) 3 (2001) 39-92.

[2] L. Ambrosio, N. Fusco and D. Pallara, Functions of Bounded Variation and Free Discontinuity Problems. Oxford Mathematical Monographs. Oxford University Press (2000).

[3] T.M. Apostol, Mathematical analysis; a modern approach to advanced calculus. Addison-Wesley Pub. Co., Reading, Mass. (1957).

[4] H. Attouch, G. Buttazzo and G. Michaille, Variational analysis in Sobolev and BV spaces. Vol. 6 of MPS/SIAM Series on Optimization, Society for Industrial and Applied Mathematics (SIAM). Applications to PDEs and optimization. Mathematical Programming Society (MPS), Philadelphia, PA (2006).

[5] L. Baratchart, D. Hardin, E. Lima, E. Saff and B. Weiss, Characterizing kernels of operators related to thin-plate magnetizations via generalizations of Hodge decompositions. Inverse Probl. 29 (2013) 015004.

[6] L. Baratchart, D. Hardin and C. Villalobos-Guillén, Divergence-free measures in the plane and inverse potential problems in divergence form. Preprint arXiv:2006.09072v2 (2020).

[7] L. Baratchart, C. Villalobos Guillén, D.P. Hardin, M.C. Northington and E.B. Saff, Inverse potential problems for divergence of measures with total variation regularization. Foundations of Computational Mathematics (2019).

[8] P. Bonicatto and N.A. Gusev, On the structure of divergence-free measures on $\mathbb{R}^{2}$. Preprint: arXiv:1912.10936 (2019).

[9] K. Bredies and M. Carioni, Sparsity of solutions for variational inverse problems with finite-dimensional data. Calc. Var. 59 (2020).

[10] K. Bredies and H.K. Pikkarainen, Inverse problems in spaces of measures. ESAIM: COCV 19 (2013) $190-218$.

[11] M. Burger and S. Osher, Convergence rates of convex variational regularization. Inverse Probl. 20 (2004) $1411-1421$.

[12] E. De Giorgi, Complementi alla teoria della misura $(n-1)$-dimensionale in uno spazio $n$-dimensionale, Seminario di Matematica della Scuola Normale Superiore di Pisa, 1960-61, Editrice Tecnico Scientifica, Pisa (1961).

[13] E. De Giorgi, Frontiere orientate di misura minima, Seminario di Matematica della Scuola Normale Superiore di Pisa, 1960-61, Editrice Tecnico Scientifica, Pisa (1961).

[14] F. Demengel and G. Demengel, Functional spaces for the theory of elliptic partial differential equations. Translated from the 2007 French original by Reinie Erné. Universitext, Springer, London; EDP Sciences, Les Ulis (2012).

[15] L.C. Evans and R.F. Gariepy, Measure Theory and Fine Properties of Functions. CRC Press (2015).

[16] H. Federer, The Gauss-Green theorem. Trans. Am. Math. Soc. 58 (1945) 44-76.

[17] H. Federer, A note on the Gauss-Green theorem. Proc. Am. Math. Soc. 9 (1958) 447-451.

[18] H. Federer, Geometric measure theory. Die Grundlehren der mathematischen Wissenschaften, Band 153. Springer-Verlag New York Inc., New York (1969).

[19] S. Foucart and H. Rauhut, A Mathematical Introduction to Compressive Sensing. Birkhäuser (2013).

[20] C. Gerhards, On the unique reconstruction of induced spherical magnetizations. Inverse Probl. 32 (2015).

[21] B. Hoffmann, B. Kaltenbacher, C. Pöschl and O. Scherzer, A convergence rates result for Tikhonov regularization in Banach spaces with non-smooth operators. Inverse Probl. 23 (2007) 987-1010.

[22] J.D. Jackson, Classical electrodynamics, John Wiley \& Sons, Inc., New York-London-Sydney (1975), second ed.

[23] J.R. Kirtley and J.P. Wikswo, Scanning squid microscopy. Annu. Rev. Mater. Sci. 29 (1999) 117-148.

[24] E.A. Lima, B.P. Weiss, L. Baratchart, D.P. Hardin and E.B. Saff, Fast inversion of magnetic field maps of unidirectional planar geological magnetization. J. Geophys Res. 118 (2013) 2723-2752.

[25] P. Mattila, Geometry of sets and measures in Euclidean spaces. Fractals and rectifiability. Vol. 44 of Cambridge Studies in Advanced Mathematics. Cambridge University Press, Cambridge (1995).

[26] W. Rudin, Real and Complex Analysis. Mc Graw-Hill (1986). 
[27] L. Schwartz, Théorie des distributions. Tome I, Actualités Sci. Ind., no. 1091 = Publ. Inst. Math. Univ. Strasbourg 9, Hermann \& Cie., Paris (1950).

[28] S.K. Smirnov, Decomposition of solenoidal vector charges into elementary solenoids, and the structure of normal onedimensional flows. St. Petersburg Math. J. 5 (1994) 841-867.

[29] C. Villalobos Guillén, A Measure Theoretic Approach for the Recovery of Remanent Magnetizations. Ph.D. thesis, Vanderbilt University (2019).

[30] B.P. Weiss, E.A. Lima, L.E. Fong and F.J. Baudenbacher, Paleomagnetic analysis using squid microscopy. J. Geophys Res. 112 (2007).

[31] W.P. Ziemer, Weakly differentiable functions. Vol. 120 of Graduate Texts in Mathematics. Sobolev spaces and functions of bounded variation. Springer-Verlag, New York (1989).

\section{Subscribe to Open (S2O) A fair and sustainable open access model}

This journal is currently published in open access with no charge for authors under a Subscribe-to-Open model $(\mathrm{S} 2 \mathrm{O})$. Open access is the free, immediate, online availability of research articles combined with the rights to use these articles fully in the digital environment.

$\mathrm{S} 2 \mathrm{O}$ is one of the transformative models that aim to move subscription journals to open access. Every year, as long as the minimum amount of subscriptions necessary to sustain the publication of the journal is attained, the content for the year is published in open access.

\section{Ask your library to support open access by subscribing to this S2O journal.}

Please help to maintain this journal in open access! Encourage your library to subscribe or verify its subscription by contacting subscribers@edpsciences.org

We are thankful to our subscribers and sponsors for making it possible to publish the journal in open access, free of charge for authors. More information and list of sponsors: https://www.edpsciences.org/en/maths-s2o-programme 